\title{
A priori estimates for the Yamabe problem in the non-locally conformally flat case
}

Fernando C. Marques 
Abstract. Given a compact Riemannian manifold $\left(M^{n}, g\right)$, with positive Yamabe quotient, not conformally diffeomorphic to the standard sphere, we prove a priori estimates for solutions to the Yamabe problem. We restrict ourselves to the dimensions where the Positive Mass Theorem is known to be true, that is, when $n \leq 7$. We also show that, when $n \geq 6$, the Weyl tensor has to vanish at a point where solutions to the Yamabe equation blow up. 


\section{Contents}

1. Introduction 4

$\begin{array}{ll}\text { 2. A Pohozaev-tvpe identity } & 7\end{array}$

3. Conformal scalar curvature equation $\quad 9$

4. Isolated and isolated simple blowup points 11

5. Svmmetrv estimates 21

6. Local blowup analvsis 28

7. Compactness theorem 35

Bibliography 39 


\section{Introduction}

Let $\left(M^{n}, g\right)$ be a compact Riemannian n-manifold, $n \geq 3$, without boundary. The classical Yamabe problem consists of finding a metric $\tilde{g}$, conformally related to $g$, with constant scalar curvature on $M$. It can be considered as a generalization of the classical Uniformization Theorem on Riemann surfaces to the setting of higher dimensional manifolds.

In analytical terms, the problem is equivalent to show the existence of a positive solution $u$ to the equation

$$
\Delta_{g} u-\frac{n-2}{4(n-1)} R_{g} u+K u^{\frac{n+2}{n-2}}=0 \text { on } M,
$$

where $\Delta_{g}$ denotes the Laplace-Beltrami operator associated with the metric $g, R_{g}$ denotes the scalar curvature of the metric $g$, and $K$ is a constant. The linear operator $L_{g}=\Delta_{g}-\frac{n-2}{4(n-1)} R_{g}$ is called the conformal Laplacian of the metric $g$.

The solution of the Yamabe problem was an outstanding achievement since, for the first time, it was given a very satisfactory existence theory to a nonlinear partial differential equation involving a critical exponent. After the initial paper in 1960 by Yamabe [19, which contained an error, contributions made by Trudinger [18, Aubin [1], and finally by Schoen [15] in 1984, solved the problem completely in the affirmative.

In this paper we shall be interested in the set of solutions to the Yamabe problem. When the first eigenvalue of the conformal Laplacian $L_{g}$ is negative, it is not difficult to see that the solution is unique. If this eigenvalue is zero, the equation becomes linear and then solutions are unique up to a constant. Therefore the only interesting case left is the positive one.

We know that when the underlying manifold is the sphere $\left(\mathbb{S}^{n}, g_{0}\right)$, endowed with the standard metric, M. Obata's theorem (see 11]) classifies all solutions to the equation, and this set is noncompact in the $C^{2}$ topology. On the other hand, the standard sphere is the only compact manifold with a noncompact group of conformal diffeomorphisms, so one should expect different behavior in the other cases.

In 13, R. Schoen proved the compactness, in the $C^{2}$ topology, of the set of solutions to the Yamabe equation, in the positive case, for every locally conformally flat manifold not conformally diffeomorphic to the sphere. He also suggested a strategy to prove these a priori $C^{2, \alpha}$ 
estimates in the non-locally conformally flat case, based on Pohozaevtype identities. In 9, Y. Y. Li and M. Zhu followed these lines and proved the theorem in dimension 3, in which case standard estimates on the blowing up solutions are sufficient for a Pohozaev identity to be applied. The compactness result in dimensions 4 and 5 was obtained by O. Druet in [4, 5]. Other compactness theorems for the Yamabe equation, in the locally conformally flat case, are proved in [6] for manifolds with boundary, and in $\mathbf{1 2}$ for singular solutions on the sphere.

Our main result in this paper is the following a priori estimates theorem in the general case:

THEOREM 1.1. Let $\left(M^{n}, g\right)$ be a smooth closed Riemannian manifold with positive Yamabe quotient, not conformally equivalent to $\left(\mathbb{S}^{n}, g_{0}\right)$. Assume $n \leq 7$. Then, for every $\epsilon>0$, there exists a positive constant $C=C(\epsilon, g)$ so that

$$
\left\{\begin{array}{r}
1 / C \leq u \leq C \text { and } \\
\|u\|_{C^{2, \alpha}(M)} \leq C
\end{array}\right.
$$

for every $u \in \cup_{1+\epsilon \leq p \leq \frac{n+2}{n-2}} \mathbb{M}_{p}$, where $0<\alpha<1$ and

$$
\mathbb{M}_{p}=\left\{u>0: \Delta_{g} u-\frac{n-2}{4(n-1)} R_{g} u+K u^{p}=0 \text { on } M\right\} .
$$

These estimates clearly imply the compactness of the set of solutions to the Yamabe equation in the $C^{2}$ topology. We restrict ourselves to the dimensions covered by the Positive Mass Theorem due to Schoen and Yau [16], i.e., $n \leq 7$, since the final global argument in our proof depends essentially on this result. Due to technical problems concerning singularities of minimizing hypersurfaces, the Positive Mass Theorem is still not known to be true for dimensions greater than 7 .

Our result will follow from a contradiction between a local restriction coming from a Pohozaev-type identity and a global argument provided by the Positive Mass Theorem. In order to accomplish that we will need a careful blowup analysis of solutions, part of it inspired by the work of C. C. Chen and C. S. Lin 3. We introduce some new symmetry estimates which allow us to control how close the blowing up solutions get to some specific rotationally symmetric functions. These symmetric functions will be solutions to the corresponding critical $\left(p=\frac{n+2}{n-2}\right)$ equation in $\mathbb{R}^{n}$. Since we are also dealing with subcritical equations, estimates on $\tau=\frac{n+2}{n-2}-p$ are also given. 
We would like to point out that the symmetry estimates (Proposition 5.4), when $n \leq 5$, are just as in the conformally flat case. However, when $n \geq 6$ we can no longer expect these same estimates to hold. This is because, in general, the asymptotic expansion of the Green function for the conformal Laplacian has additional terms (see [7).

One important difficulty we must overcome when $n \geq 6$, pointed out by Schoen in [13, is to show conformal flatness of the metric to a sufficiently high order at a blowup point. This is needed in order to apply the Positive Mass Theorem when $n=6$ or 7 . That is the content of our next theorem, where $W_{g}$ denotes the Weyl tensor of the metric $g$ :

TheOrem 1.2. Assume $n \geq 6$ and let $u_{i}$ be a sequence of positive solutions to (1.1). Suppose $x_{i} \rightarrow \bar{x}$ is a sequence of points such that $u_{i}\left(x_{i}\right) \rightarrow \infty$ as $i \rightarrow \infty$. Then

$$
W_{g}(\bar{x})=0 .
$$

In general one should expect, as indicated by Schoen in 14, that at a blowup point $\bar{x}$ we must have :

$$
\nabla^{k} W_{g}(\bar{x})=0
$$

for all $0 \leq k \leq \frac{n-6}{2}$. The proof of Theorem 1.2 also relies upon the symmetry estimates and a Pohozaev-type identity.

It is not difficult to check that the Theorem 1.1 implies the existence of a solution to the Yamabe problem. This is because solutions $u_{i}$ to the subcritical equations, with $p_{i} \rightarrow \frac{n+2}{n-2}$ as $i \rightarrow \infty$, can be constructed by standard variational methods. Actually one can say more. Another consequence of the compactness theorem is the computation of the total Leray-Schauder degree of all solutions to equation (1.1):

THEOREM 1.3. Let $\left(M^{n}, g\right)$ be a smooth closed Riemannian manifold with positive Yamabe quotient, not conformally equivalent to $\left(\mathbb{S}^{n}, g_{0}\right)$, $4 \leq n \leq 7$. Then, if $\Lambda$ is sufficiently large,

$$
\begin{gathered}
\operatorname{deg}\left(F, \Omega_{\Lambda}, 0\right)=-1, \\
\text { where } F(u)=u+L_{g}^{-1}\left(E(u) u^{\frac{n+2}{n-2}}\right), E(u)=-\int_{M} u L_{g}(u) d v_{g} \text { and } \\
\Omega_{\Lambda}=\left\{u \in C^{2, \alpha}(M): \min _{M} u>\Lambda^{-1},\|u\|_{2, \alpha}<\Lambda\right\} .
\end{gathered}
$$

This theorem follows from Theorem 1.1 and arguments given by Schoen in [13. 
The author was just recently communicated that Y. Y. Li and L. Zhang have independently proved the same compactness result.

Acknowledgements. The content of this paper is part of the author's doctoral thesis [10. The author would like to dedicate this paper to the memory of his friend and advisor Prof. José F. Escobar. His encouragement was invaluable for the completion of this work. While the author was at Cornell University, he was fully supported by CNPqBrazil.

\section{A Pohozaev-type identity}

In this section we will establish a Pohozaev-type identity which will be very useful in the subsequent blowup analysis.

Suppose $u: B_{\rho}(0) \backslash\{0\} \subset \mathbb{R}^{n} \rightarrow \mathbb{R}$ is a positive $C^{2}$ solution to the equation

$$
a^{i j}(x) \partial_{i j} u+b^{i}(x) \partial_{i} u+c(x) u+K(x) u^{p}=0,
$$

where $p \neq-1, K \in C^{1}$ and $a^{i j}, b^{i}, c$ are continuous functions, $1 \leq i, j \leq$ $n$. Here we are using the summation convention.

Define

$$
\int_{|x|=r}\left(\frac{n-2}{2} u \frac{\partial u}{\partial r}-\frac{|x|}{2}|\nabla u|^{2}+|x|\left|\frac{\partial u}{\partial r}\right|^{2}+\frac{1}{p+1} K(x)|x| u^{p+1}\right) d \sigma(r),
$$

whenever $0<r<\rho$.

The following lemma gives the Pohozaev-type identity we are interested in.

Lemma 2.1. Given $0<s \leq r<\rho$,

$$
\begin{gathered}
P(r, u)-P(s, u)=-\int_{s \leq|x| \leq r}\left(x^{k} \partial_{k} u+\frac{n-2}{2} u\right) A(u) d x \\
+\frac{1}{p+1} \int_{s \leq|x| \leq r}\left(x^{k} \partial_{k} K(x)\right) u^{p+1} d x \\
+\left(\frac{n}{p+1}-\frac{n-2}{2}\right) \int_{s \leq|x| \leq r} K(x) u^{p+1} d x,
\end{gathered}
$$

where $A(u)=\left(a^{i j}-\delta_{i j}\right) \partial_{i j} u+b^{i} \partial_{i} u+c u$. 
Proof. Multiplying the equation (2.1) by $x^{k} \partial_{k} u$, and integrating over the set $\{x: s \leq|x| \leq r\}$, we obtain

$$
\int_{s \leq|x| \leq r}\left(x^{k} \partial_{k} u\right)\left(\Delta u+A(u)+K(x) u^{p}\right) d x=0 .
$$

Integration by parts gives:

$$
\begin{aligned}
& \int_{s \leq|x| \leq r}\left(x^{k} \partial_{k} u\right) \partial_{i i} u d x= \\
& -\int_{s \leq|x| \leq r}\left(\delta_{i}^{k} \partial_{k} u \partial_{i} u+\frac{1}{2} x^{k} \partial_{k}\left[\left(\partial_{i} u\right)^{2}\right]\right) d x \\
& \quad+\frac{1}{r} \int_{|x|=r}\left(x^{k} \partial_{k} u\right)\left(x_{i} \partial_{i} u\right)-\frac{1}{s} \int_{|x|=s}\left(x^{k} \partial_{k} u\right)\left(x_{i} \partial_{i} u\right)= \\
& -\int_{s \leq|x| \leq r}\left(\delta_{i}^{k} \partial_{k} u \partial_{i} u-\frac{n}{2}\left(\partial_{i} u\right)^{2}\right) d x-\frac{r}{2} \int_{|x|=r}\left(\partial_{i} u\right)^{2}+\frac{s}{2} \int_{|x|=s}\left(\partial_{i} u\right)^{2} \\
& \quad+\frac{1}{r} \int_{|x|=r}\left(x^{k} \partial_{k} u\right)\left(x_{i} \partial_{i} u\right)-\frac{1}{s} \int_{|x|=s}\left(x^{k} \partial_{k} u\right)\left(x_{i} \partial_{i} u\right),
\end{aligned}
$$

and summing over $i=1, \ldots, n$, we obtain

$$
\begin{aligned}
& \int_{s \leq|x| \leq r}\left(x^{k} \partial_{k} u\right) \Delta u d x= \\
& \frac{n-2}{2} \int_{s \leq|x| \leq r}|\nabla u|^{2} d x-\frac{r}{2} \int_{|x|=r}|\nabla u|^{2}+\frac{s}{2} \int_{|x|=s}|\nabla u|^{2} \\
& \quad+r \int_{|x|=r}\left(\frac{\partial u}{\partial r}\right)^{2}-s \int_{|x|=s}\left(\frac{\partial u}{\partial r}\right)^{2} .
\end{aligned}
$$

Also

$$
\begin{aligned}
& \int_{s \leq|x| \leq r}\left(x^{k} \partial_{k} u\right) K(x) u^{p} d x= \\
& \frac{1}{p+1} \int_{s \leq|x| \leq r} x^{k} \partial_{k}\left(u^{p+1}\right) K(x) d x= \\
& -\frac{n}{p+1} \int_{s \leq|x| \leq r} K(x) u^{p+1} d x-\frac{1}{p+1} \int_{s \leq|x| \leq r}\left(x^{k} \partial_{k} K(x)\right) u^{p+1} d x \\
& \quad+\frac{r}{p+1} \int_{|x|=r} K(x) u^{p+1}-\frac{s}{p+1} \int_{|x|=s} K(x) u^{p+1} .
\end{aligned}
$$


On the other hand, multiplying the equation (2.1) by $\mathrm{u}$, and once again integrating by parts, we get

$$
\begin{aligned}
& \int_{s \leq|x| \leq r}|\nabla u|^{2} d x= \\
& \quad \int_{s \leq|x| \leq r}\left(u A(u)+K(x) u^{p+1}\right) d x+\int_{|x|=r} u \frac{\partial u}{\partial r}-\int_{|x|=s} u \frac{\partial u}{\partial r} .
\end{aligned}
$$

Now we substitute equalities (2.4), (2.5) and equality (2.6) in equality (2.3) and the Pohozaev identity follows by rearranging terms. q.e.d.

When $\mathrm{u}$ is a solution to the equation (2.1) in the entire ball, by taking the limit as $s \rightarrow 0$ we get

$$
\begin{gathered}
P(r, u)=-\int_{|x| \leq r}\left(x^{k} \partial_{k} u+\frac{n-2}{2} u\right) A(u) d x \\
+\frac{1}{p+1} \int_{|x| \leq r}\left(x^{k} \partial_{k} K(x)\right) u^{p+1} d x+ \\
\left(\frac{n}{p+1}-\frac{n-2}{2}\right) \int_{|x| \leq r} K(x) u^{p+1} d x .
\end{gathered}
$$

Integrating by parts once more, we can also get

$$
\begin{aligned}
& P(r, u)=-\int_{|x| \leq r}\left(x^{k} \partial_{k} u+\frac{n-2}{2} u\right)\left(\left(a^{i j}-\delta^{i j}\right) \partial_{i j} u+b^{i} \partial_{i} u\right) d x \\
& \quad+\int_{|x| \leq r}\left(\frac{1}{2} x^{k} \partial_{k} c+c\right) u^{2} d x-\frac{r}{2} \int_{|x|=r} c u^{2} d \sigma(r) \\
& \quad+\frac{1}{p+1} \int_{|x| \leq r}\left(x^{k} \partial_{k} K(x)\right) u^{p+1} d x \\
& +\left(\frac{n}{p+1}-\frac{n-2}{2}\right) \int_{|x| \leq r} K(x) u^{p+1} d x
\end{aligned}
$$

\section{Conformal scalar curvature equation}

In this section we will introduce the partial differential equation we are interested in, and we shall discuss some of its properties related to conformal deformation of metrics.

Let $\Omega \in \mathbb{R}^{n}$ be an open set, and suppose $g$ is a Riemannian metric in $\Omega$. Suppose also $f$ is a positive $C^{1}$ function defined in $\Omega$.

Consider a positive $C^{2}$ function $u$ satisfying

$$
\Delta_{g} u-c(n) R_{g} u+K f^{-\tau} u^{p}=0 \text { in } \Omega,
$$


where $c(n)=\frac{n-2}{4(n-1)}, K=n(n-2), 1<p \leq \frac{n+2}{n-2}$ and $\tau=\frac{n+2}{n-2}-p$. We will use the notation $R_{g}$ for the scalar curvature of $g$. The operator $L_{g}=\Delta_{g}-c(n) R_{g}$ is called the conformal Laplacian of the metric $g$.

When $p=\frac{n+2}{n-2}$, this partial differential equation is intimately related to conformal geometry, particularly when one studies conformally related metrics with constant scalar curvature. More specifically, given a positive solution $u$, the metric $u^{\frac{4}{n-2}} g$ has constant scalar curvature equal to $4 n(n-1)$.

Now let us describe an important feature of solutions to that type of equation. Let $u$ be a solution to equation (3.1) and choose $\bar{x} \in \Omega$. Given $s>0$, define the renormalized function

$$
v(y)=s^{\frac{2}{p-1}} u(\exp \bar{x}(s y)) .
$$

Then

$$
L_{h} v+K \tilde{f}^{-\tau} v^{p}=0,
$$

where $\tilde{f}(y)=f(s y)$ and the components of the metric $h$ in normal coordinates are given by $h_{k l}(y)=g_{k l}(s y)$. The important point here is that $v$ satisfies an equation of the same type.

The equation is also conformally invariant in the following sense. Suppose $\tilde{g}=\phi^{\frac{4}{n-2}} g$ is a metric conformal to $g$. Let us recall

$$
L_{\tilde{g}}\left(\phi^{-1} u\right)=\phi^{-\frac{n+2}{n-2}} L_{g}(u)
$$

for any function $u$, and

$$
R_{\tilde{g}}=-c(n)^{-1} \phi^{-\frac{n+2}{n-2}} L_{g}(\phi) .
$$

(See 7).

Therefore, if $u$ is a solution to (3.1), then $\phi^{-1} u$ satisfies

$$
L_{\tilde{g}}\left(\phi^{-1} u\right)+K(\phi f)^{-\tau}\left(\phi^{-1} u\right)^{p}=0,
$$

which is again an equation of the same type.

This will have very important consequences in what follows. We will study sequences of solutions $u_{i}$ to equation (3.1). When what we want to study is conformally invariant, we are allowed to replace $u_{i}$ by another sequence of functions $v_{i}=\phi_{i}^{-1} u_{i}$, at the same time replacing the metric $g_{i}$ by $\tilde{g}_{i}=\phi_{i}^{\frac{4}{n-2}} g_{i}$, as long as we have a uniform control on the conformal factors $\phi_{i}$. In this paper, there will be two examples of such a procedure. 
First, we can suppose the metric $g_{i}$ has positive scalar curvature in a small ball centered at some fixed point $x_{i}$. To see this, fix $\sigma>0$ small and let $\phi$ be the first eigenfunction of $\Delta_{g}$ with respect to the Dirichlet condition:

$$
\left\{\begin{array}{r}
\Delta_{g} \phi+\lambda_{1} \phi=0 \text { in } B_{2 \sigma}(x) \\
\phi=0 \text { on } \partial B_{2 \sigma}(x)
\end{array}\right.
$$

Recall that the corresponding eigenspace is one-dimensional and we can choose $\phi>0$ on $B_{2 \sigma}(x)$. Now, since $\lambda_{1} \rightarrow \infty$ as $\sigma \rightarrow 0$, we can choose $\sigma$ small enough so that

$$
\Delta_{g} \phi-c(n) R_{g} \phi<0
$$

in $B_{\sigma}(x)$. Defining $\tilde{g}=\phi^{\frac{4}{n-2}} g$, relation (3.3) implies $R_{\tilde{g}}>0$ in $B_{\sigma}(x)$. Moreover, if we take as a conformal factor the solution $\psi$ to:

$$
\left\{\begin{array}{r}
\Delta_{g} \psi-c(n) R_{g} \psi=0 \text { in } B_{\sigma}(x) \\
\psi=1 \text { on } \partial B_{\sigma}(x),
\end{array}\right.
$$

we can also have $R_{\tilde{g}}=0$.

The second example is related to the so-called conformal normal coordinates. (See $\mathbf{7}$ ). Given an integer $N \geq 2$, there exists a positive function $\phi$ (which can be constructed explicitly), such that, setting $\tilde{g}=$ $\phi^{\frac{4}{n-2}} g$, the volume element satisfies:

$$
\operatorname{det}\left(\tilde{g}_{i j}\right)=1+O\left(r^{N}\right),
$$

in $\tilde{g}$-normal coordinates around $x$, where $r=d_{\tilde{g}}(x, \cdot)$. This allows us to simplify the local asymptotic analysis. For example, in conformal normal coordinates around $x, R_{\tilde{g}}=O\left(r^{2}\right)$ and $\Delta R_{\tilde{g}}(x)=-\frac{1}{6}\left|W_{\tilde{g}}(x)\right|^{2}$, where $W$ stands for the Weyl tensor.

\section{Isolated and isolated simple blowup points}

In this section we will define isolated and isolated simple blowup points and we shall discuss their basic properties. The results in this section are well-known in the locally conformally flat setting $(\mathbf{8}, \mathbf{1 7}$ ) and in general when $n=3([\mathbf{9})$. We will slightly modify their proofs in 9 to make them work in any dimension.

Let $\Omega \subset \mathbb{R}^{n}$ be an open set, and suppose $g_{i}$ is a sequence of Riemannian metrics in $\Omega$ converging, in the $C_{l o c}^{2}$ topology, to a metric g. Suppose also that $f_{i}$ is a sequence of positive $C^{1}$ functions converging in the $C_{l o c}^{1}$ topology to a positive function $f$. 
We will consider a sequence $u_{i}$ of positive $C^{2}$ functions satisfying

$$
L_{g_{i}} u_{i}+K f_{i}^{-\tau_{i}} u_{i}^{p_{i}}=0 \text { in } \Omega \text {, }
$$

where $c(n)=\frac{n-2}{4(n-1)}, K=n(n-2), 1+\epsilon_{0}<p_{i} \leq \frac{n+2}{n-2}$ for some $\epsilon_{0}>0$ and $\tau_{i}=\frac{n+2}{n-2}-p_{i}$.

We will sometimes omit the subscript $i$, for the sake of simplicity, and we will use the symbols $c, C$ to denote various positive constants.

Definition 4.1. We say that $\bar{x} \in \Omega$ is an isolated blowup point for $u_{i}$ if there exists a sequence $x_{i} \in \Omega$, converging to $\bar{x}$, so that:

(1) $x_{i}$ is a local maximum point of $u_{i}$;

(2) $M_{i}:=u_{i}\left(x_{i}\right) \rightarrow \infty$ as $i \rightarrow \infty$;

(3) there exist $r, C>0$ such that

$$
u_{i}(x) \leq C d_{g_{i}}\left(x, x_{i}\right)^{-\frac{2}{p_{i}-1}}
$$

for every $x \in B_{r}\left(x_{i}\right) \subset \Omega$. Here $B_{r}\left(x_{i}\right)$ denotes the geodesic ball of radius $r$, centered at $x_{i}$, with respect to the metric $g_{i}$.

Remark: In various parts of the text, we will identify $x_{i}$ with the origin, that meaning we are making use of normal coordinates in a small ball around $x_{i}$. More precisely, we will sometimes write $u_{i}(x)$ instead of $u_{i}\left(\exp _{x_{i}}(x)\right)$ and $|x|$ instead of $d_{g_{i}}\left(x, x_{i}\right)$, and those functions will be defined in balls centered at 0 .

Note that the definition of isolated blowup points is invariant under renormalization, which was described in the last section. This follows from the fact that, if $v(y)=s^{\frac{2}{p_{i}-1}} u(s y)$, then

$$
u(x) \leq C|x|^{-\frac{2}{p_{i}-1}} \Leftrightarrow v(y) \leq C|y|^{-\frac{2}{p_{i}-1}} .
$$

The first result concerning isolated blowup points is the following Harnack inequality.

Proposition 4.2. Suppose that $u_{i}$ is a sequence of positive functions satisfying equation (4.1) and assume $x_{i} \rightarrow \bar{x}$ is an isolated blowup point. Then there exists a constant $C>0$ such that

$$
\max _{\frac{s}{2} \leq d_{g_{i}}\left(x, x_{i}\right) \leq 2 s} u_{i}(x) \leq C \min _{\frac{s}{2} \leq d_{g_{i}}\left(x, x_{i}\right) \leq 2 s} u_{i}(x),
$$

where $0<s<\frac{r}{3}$. 
Proof. Let $\left(x^{1}, \ldots, x^{n}\right)$ be normal coordinates with respect to the metric $g_{i}$ on the ball $B_{r}\left(x_{i}\right)$. (See remark after Definition 4.1).

Define

$$
v_{i}(y)=s^{\frac{2}{p_{i}-1}} u_{i}(s y),
$$

where $|y|<3$.

Then, as discussed in the last section,

$$
L_{h_{i}} v_{i}(y)+K \tilde{f}_{i}^{-\tau_{i}} v_{i}^{p_{i}}(y)=0,
$$

where $\tilde{f}_{i}(y)=f_{i}(s y)$ and $\left(h_{i}\right)_{k l}(y)=\left(g_{i}\right)_{k l}(s y)$, and we also know that

$$
v_{i}(y) \leq C|y|^{-\frac{2}{p_{i}-1}},
$$

whenever $|y|<3$.

It follows from this last inequality that $v_{i}$ is uniformly bounded in compact subsets of $B_{3}(0) \backslash\{0\}$. The Harnack inequality for elliptic linear equations then implies that there exists $C>0$ such that

$$
\max _{\frac{1}{2} \leq|y| \leq 2} v_{i}(y) \leq C \min _{\frac{1}{2} \leq|y| \leq 2} v_{i}(y) .
$$

The result now follows directly.

q.e.d.

The Proposition 4.2 clearly implies the so-called spherical Harnack inequality for isolated blowup points. Namely, given $0<s \leq \frac{2}{3} r$, there exists a positive constant $C$, not depending on $s$, such that

$$
\max _{d_{g_{i}}\left(x, x_{i}\right)=s} u_{i}(x) \leq C \min _{d_{g_{i}}\left(x, x_{i}\right)=s} u_{i}(x) .
$$

Define $U_{0}(y)=\left(1+|y|^{2}\right)^{\frac{2-n}{2}}$. It is not difficult to check that

$$
\Delta U_{0}(y)+K U_{0}^{\frac{n+2}{n-2}}(y)=0 .
$$

The next proposition says that, in the case of an isolated blowup point, the functions $u_{i}$, when renormalized, converge in the $C^{2}$ topology to the rotationally symmetric function $U_{0}$.

Proposition 4.3. Let $u_{i}$ be a sequence of positive functions satisfying the equation (4.1) and $x_{i} \rightarrow \bar{x}$ be an isolated blowup point. Assume that $R_{i} \rightarrow \infty$ and $\epsilon_{i} \rightarrow 0$ are given. Then $p_{i} \rightarrow \frac{n+2}{n-2}$ and, after possibly passing to a subsequence,

$$
\left\|M_{i}^{-1} u_{i}\left(M_{i}^{-\frac{p_{i}-1}{2}} y\right)-U_{0}(y)\right\|_{C^{2}\left(B_{R_{i}}(0)\right)} \leq \epsilon_{i}
$$


and

$$
\frac{R_{i}}{\log M_{i}} \rightarrow 0 \text { as } i \rightarrow \infty
$$

Here $M_{i}$ is as in Definition 4.1 .

Proof. Let $\left(x^{1}, \ldots, x^{n}\right)$ be normal coordinates with respect to the metric $g_{i}$ on the ball $B_{r}\left(x_{i}\right)$. (See first remark after Definition 4.1).

Define

$$
v_{i}(y)=M_{i}^{-1} u_{i}\left(M_{i}^{-\frac{p_{i}-1}{2}} y\right)
$$

for $|y|<r M_{i}^{\frac{p_{i}-1}{2}}$. Here $r$ is as in Definition 4.1.

Then

$$
L_{h_{i}} v_{i}(y)+K \tilde{f}_{i}^{-\tau_{i}} v_{i}^{p_{i}}(y)=0,
$$

where $\tilde{f}_{i}(y)=f_{i}\left(M_{i}^{-\frac{p_{i}-1}{2}} y\right)$ and $\left(h_{i}\right)_{k l}(y)=\left(g_{i}\right)_{k l}\left(M_{i}^{-\frac{p_{i}-1}{2}} y\right)$.

Note also that

$$
\left\{\begin{array}{r}
v_{i}(0)=1, \nabla v_{i}(0)=0 \\
0<v_{i}(y) \leq C|y|^{-\frac{2}{p_{i}-1}} \text { for }|y|<r M_{i}^{\frac{p_{i}-1}{2}} .
\end{array}\right.
$$

Claim: There exists $C>0$ such that $v_{i}(y) \leq C$, whenever $|y|<$ $r M_{i}^{\frac{p_{i}-1}{2}}$.

Proof of Claim: From properties (4.5), we get

$$
v_{i}(y) \leq C,
$$

if $1 \leq|y| \leq r M_{i}^{\frac{p_{i}-1}{2}}$.

Now, from what was discussed in the previous section, up to a conformal deformation we can suppose our metrics have zero scalar curvature in small balls. In particular their conformal Laplacians will satisfy the maximum principle. This implies there exists $C>0$ so that

$$
\min _{|y| \leq r} v_{i}(y) \geq C^{-1} \min _{|y|=r} v_{i}(y) \quad \forall i
$$

and $0<r \leq 1$. The spherical Harnack inequality (4.3) implies

$$
\begin{aligned}
\max _{|y|=r} v_{i}(y) & \leq C \min _{|y|=r} v_{i}(y) \leq C \min _{|y| \leq r} v_{i}(y) \\
& \leq C v_{i}(0)=C
\end{aligned}
$$

for $0<r \leq 1$. This and inequality (4.6) imply the claim. 
Standard elliptic estimates now imply that, after passing to a subsequence, $v_{i} \rightarrow v>0$ in $C_{l o c}^{2}\left(\mathbb{R}^{n}\right)$, where

$$
\left\{\begin{array}{r}
\Delta v(y)+K v^{p}(y)=0, y \in \mathbb{R}^{n} \\
v(0)=1, \quad \nabla v(0)=0
\end{array}\right.
$$

where $p=\lim _{i \rightarrow \infty} p_{i}$. Here $\Delta$ denotes the Euclidean Laplacian.

A well-known theorem by Caffarelli, Gidas and Spruck [2], states that we necessarily have $p=\frac{n+2}{n-2}$ and $v(y)=U_{0}(y)$.

The Proposition now follows easily.

q.e.d.

Now let us introduce the notion of an isolated simple blowup point.

Suppose $u_{i}$ is a sequence of positive functions satisfying equation (4.1) and $x_{i} \rightarrow \bar{x}$ is an isolated blowup point. Define

$$
\bar{u}_{i}(r)=\frac{1}{\sigma_{n-1} r^{n-1}} \int_{\partial B_{r}\left(x_{i}\right)} u_{i} d \sigma(r)
$$

where $\sigma_{n-1}$ denotes the area of a unit sphere in $\mathbb{R}^{n}$. We are using $g_{i}$-normal coordinates and integrating with respect to the Euclidean volume form.

Definition 4.4. We say $x_{i} \rightarrow \bar{x}$ is an isolated simple blowup point if there exists a real number $0<\rho<r$ such that the functions

$$
\hat{u}_{i}(r)=r^{\frac{2}{p_{i}-1}} \bar{u}_{i}(r)
$$

have exactly one critical point in the interval $(0, \rho)$, for $i$ large.

It is not difficult to see that Proposition 4.3 implies that $\hat{u}_{i}$ has exactly one critical point in the interval $\left(0, R_{i} u_{i}\left(x_{i}\right)^{-\frac{p_{i}-1}{2}}\right)$. Moreover its derivative is negative right after the critical point. As a result, if the blowup is isolated simple, then

$$
\hat{u}_{i}^{\prime}(r)<0
$$

for all $R_{i} M_{i}^{-\frac{p_{i}-1}{2}} \leq r<\rho$.

Now we turn to the first estimate on isolated simple blowup points.

Proposition 4.5. Let $u_{i}$ be a sequence of positive functions satisfying equation (4.1) and $x_{i} \rightarrow \bar{x}$ be an isolated simple blowup point. Then there exists a constant $C>0$ and $0<\rho_{1}<\rho$ such that, for each $i$,

$$
M_{i} u_{i}(x) \leq C d_{g_{i}}\left(x, x_{i}\right)^{2-n},
$$


whenever $d_{g_{i}}\left(x, x_{i}\right) \leq \rho_{1}$. Moreover, if $R_{i} M_{i}^{-\frac{p_{i}-1}{2}} \leq d_{g_{i}}\left(x, x_{i}\right) \leq \rho_{1}$, then

$$
M_{i} u_{i}(x) \geq C^{-1} G_{i}\left(x_{i}, x\right),
$$

where $G_{i}$ is the Green function of $L_{g_{i}}$ with respect to the Dirichlet boundary condition on $B_{\rho_{1}}\left(x_{i}\right)$.

Proof. We first need a slightly different estimate.

Let $\delta>0$, and define $\lambda_{i}=(n-2-\delta) \frac{p_{i}-1}{2}-1$.

Let us apply the Proposition 4.3 to some $R_{i} \rightarrow \infty$ and $0<\epsilon_{i}<e^{-R_{i}}$.

Claim 1: If $\delta$ is sufficiently small, there exist constants $0<\rho_{1}<\rho$ and $C>0$ such that

$$
\begin{aligned}
M_{i}^{\lambda_{i}} u_{i}(x) & \leq C d\left(x, x_{i}\right)^{2-n+\delta} \\
M_{i}^{\lambda_{i}}\left|\nabla u_{i}(x)\right| & \leq C d\left(x, x_{i}\right)^{1-n+\delta} \\
M_{i}^{\lambda_{i}}\left|\nabla^{2} u_{i}(x)\right| & \leq C d\left(x, x_{i}\right)^{-n+\delta}
\end{aligned}
$$

for every $x$ so that $R_{i} M_{i}^{-\frac{p_{i}-1}{2}} \leq d\left(x, x_{i}\right) \leq \rho_{1}$.

The proof of Claim 1 is analogous to the proof of the Lemma 3.3 in 9 .

Remark: It is not difficult to see that the previous estimates imply

$$
\left\{\begin{array}{l}
v_{i}(y) \quad \leq C M_{i}^{\delta \frac{p_{i}-1}{2}}(1+|y|)^{2-n} \\
\left|\nabla v_{i}(y)\right| \leq C M_{i}^{\delta \frac{p_{i}-1}{2}}(1+|y|)^{1-n} \\
\left|\nabla^{2} v_{i}(y)\right| \leq C M_{i}^{\delta \frac{p_{i}-1}{2}}(1+|y|)^{-n}
\end{array}\right.
$$

for any $|y| \leq \rho_{1} M_{i}^{\frac{p_{i}-1}{2}}$.

Let us now estimate $\tau_{i}$.

Claim 2: There exists $C>0$ such that

$$
\tau_{i} \leq\left\{\begin{array}{c}
C M_{i}^{(-1+\delta) \frac{4}{n-2}+o(1)} \text { if } n>4, \\
C M_{i}^{(-1+\delta) 2+o(1)} \log M_{i} \text { if } n=4,
\end{array}\right.
$$

and, in particular, $M_{i}^{\tau_{i}} \rightarrow 1$ as $i \rightarrow \infty$. 
Proof of Claim 2: We will apply the Pohozaev identity (2.7) from Section 2 to $u_{i}$ on the ball of radius $\frac{\rho_{1}}{2}$ :

$$
\begin{gathered}
P\left(\frac{\rho_{1}}{2}, u_{i}\right)=-\int_{|x| \leq \frac{\rho_{1}}{2}}\left(x^{m} \partial_{m} u_{i}+\frac{n-2}{2} u_{i}\right) A_{i}(x) d x \\
+\left(\frac{n}{p_{i}+1}-\frac{n-2}{2}\right) \int_{|x| \leq \frac{\rho_{1}}{2}} K f_{i}^{-\tau_{i}} u_{i}^{p_{i}+1} d x \\
-\frac{\tau_{i}}{p_{i}+1} \int_{|x| \leq \frac{\rho_{1}}{2}} K f_{i}^{-\tau_{i}-1}\left(x^{m} \partial_{m} f_{i}\right) u_{i}^{p_{i}+1} d x,
\end{gathered}
$$

where

$$
\begin{aligned}
& A_{i}(x)=\left(g^{k l}-\delta^{k l}\right)(x) \partial_{k l} u_{i}(x) \\
& \quad+\left(\partial_{k} g^{k l}+|g|^{-\frac{1}{2}} \partial_{k}\left(|g|^{\frac{1}{2}}\right) g^{k l}\right)(x) \partial_{l} u_{i}(x)-c(n) R_{g}(x) u_{i}(x)
\end{aligned}
$$

and recall

$$
\begin{aligned}
& P\left(\frac{\rho_{1}}{2}, u_{i}\right)=\int_{|x|=\frac{\rho_{1}}{2}}\left(\frac{n-2}{2} u_{i} \frac{\partial u_{i}}{\partial r}-\frac{|x|}{2}\left|\nabla u_{i}\right|^{2}+|x|\left|\frac{\partial u_{i}}{\partial r}\right|^{2}\right) d \sigma \\
& +\frac{1}{p_{i}+1} \int_{|x|=\frac{\rho_{1}}{2}} K f_{i}^{-\tau_{i}}|x| u_{i}^{p_{i}+1} d \sigma
\end{aligned}
$$

From Claim 1 we get

$$
\left|P\left(\frac{\rho_{1}}{2}, u_{i}\right)\right| \leq c M_{i}^{-2 \lambda_{i}}
$$

Define

$$
\begin{aligned}
& \hat{A}_{i}(y)=\left(g^{k l}-\delta^{k l}\right)\left(M_{i}^{-\frac{p_{i}-1}{2}} y\right) \partial_{k l} v_{i} \\
& \quad+M_{i}^{-\frac{p_{i}-1}{2}}\left(\partial_{k} g^{k l}+|g|^{-\frac{1}{2}} \partial_{k}\left(|g|^{\frac{1}{2}}\right) g^{k l}\right)\left(M_{i}^{-\frac{p_{i}-1}{2}} y\right) \partial_{l} v_{i} \\
& -c(n) M_{i}^{-\left(p_{i}-1\right)} R_{g}\left(M_{i}^{-\frac{p_{i}-1}{2}} y\right) v_{i} .
\end{aligned}
$$

The change of variables $y=M^{\frac{p_{i}-1}{2}} x$, the inequalities (4.14) and the fact that the metric is euclidean up to first order in normal coordinates 
yield

$$
\begin{gathered}
\quad\left|\int_{|x| \leq \frac{\rho_{1}}{2}}\left(x^{m} \partial_{m} u_{i}+\frac{n-2}{2} u_{i}\right) A_{i}(x) d x\right| \\
\quad=M_{i}^{p \frac{2-n}{2}} M_{i}^{\frac{n+2}{2}}\left|\int_{|y| \leq \frac{\rho_{1}}{2} M^{\frac{p_{i}-1}{2}}}\left(y^{m} \partial_{m} v_{i}+\frac{n-2}{2} v_{i}\right) \hat{A}_{i}(y) d y\right| \\
\leq C M_{i}^{p \frac{2-n}{2}} M_{i}^{\frac{n+2}{2}} M_{i}^{-\left(p_{i}-1\right)} M_{i}^{\delta\left(p_{i}-1\right)} \int_{|y| \leq \frac{\rho_{1}}{2} M^{\frac{p_{i}-1}{2}}}(1+|y|)^{4-2 n} d y \\
(4.18) \leq\left\{\begin{array}{c}
C M_{i}^{(-1+\delta) \frac{4}{n-2}+o(1)} \text { if } n>4, \\
C M_{i}^{(-1+\delta) 2+o(1)} \log M_{i} \text { if } n=4 .
\end{array}\right.
\end{gathered}
$$

Therefore, from the inequalities (4.16) and (4.18) and the identity (4.15),

$$
\begin{aligned}
& \frac{(n-2) \tau_{i}}{2\left(p_{i}+1\right)} \int_{|x| \leq \frac{\rho_{1}}{2}} K f_{i}^{-\tau_{i}} u_{i}(x)^{p_{i}+1} d x \\
& \quad-\frac{\tau_{i}}{p_{i}+1} \int_{|x| \leq \frac{\rho_{1}}{2}} K f_{i}^{-\tau_{i}-1}\left(x^{m} \partial_{m} f_{i}\right) u_{i}^{p_{i}+1} d x \\
& \leq\left\{\begin{array}{c}
C M_{i}^{(-1+\delta) \frac{4}{n-2}+o(1)} \text { if } n>4, \\
C M_{i}^{(-1+\delta) 2+o(1)} \log M_{i} \text { if } n=4 .
\end{array}\right.
\end{aligned}
$$

Since, from the Proposition 4.3 .

$$
\int_{|x| \leq R_{i} M_{i}^{-\frac{p_{i}-1}{2}}} u_{i}(x)^{p_{i}+1} d x \geq c>0
$$

we conclude that if we choose $\rho_{1}$ sufficiently small, then

$$
\begin{aligned}
& \frac{n-2}{2} \int_{|x| \leq \frac{\rho_{1}}{2}} K f_{i}^{-\tau_{i}} u_{i}(x)^{p_{i}+1} d x \\
& -\int_{|x| \leq \frac{\rho_{1}}{2}} K f_{i}^{-\tau_{i}-1}\left(x^{m} \partial_{m} f_{i}\right) u_{i}^{p_{i}+1} d x \geq c>0 .
\end{aligned}
$$

The result follows immediately from the inequalities (4.19).

Claim 3: Given a small $\sigma>0$, there exists a constant $C>0$ such that

$$
\int_{B_{\sigma}\left(x_{i}\right)} u_{i}^{p_{i}}(x) d x \leq C M_{i}^{-1} .
$$

Proof of Claim 3: Set $s_{i}=R_{i} M_{i}^{-\frac{p_{i}-1}{2}}$. 
First note that, changing variables, and then using $v_{i}(y) \leq c U_{0}(y)$ for $|y| \leq R_{i}$, we get

$$
\int_{|x| \leq s_{i}} u_{i}^{p_{i}}(x) d x=M_{i}^{-\frac{\left(p_{i}-1\right) n}{2}} M_{i}^{p_{i}} \int_{|y| \leq R_{i}} v_{i}^{p_{i}}(y) d y \leq C M_{i}^{-1} .
$$

On the other hand, by Claim 1,

$$
\begin{aligned}
\int_{s_{i} \leq|x| \leq \sigma} u_{i}^{p_{i}}(x) d x & \leq C M_{i}^{-\lambda_{i} p_{i}} \int_{s_{i} \leq|x| \leq \sigma}|x|^{(2-n+\delta) p_{i}} d x \\
& \leq C M_{i}^{-\lambda_{i} p_{i}} s_{i}^{(2-n+\delta) p_{i}+n} \leq o(1) M_{i}^{-1} .
\end{aligned}
$$

Claim 3 now follows from inequalities (4.21) and (4.22).

Claim 4: There exists $\sigma_{1}>0$ such that for all $0<\sigma<\sigma_{1}$, there exists a constant $C=C(\sigma)$ with, for every $i$,

$$
u_{i}\left(x_{i}\right) u_{i}(x) \leq C(\sigma)
$$

if $d\left(x, x_{i}\right)=\sigma$.

Proof of Claim 4: From the discussion in Section 3] if we choose $\sigma_{1}>0$ small, we can suppose that $R_{g_{i}} \geq 0$.

Choose $0<\sigma<\sigma_{1}$ small and define

$$
w_{i}(x)=u_{i}\left(x_{\sigma}\right)^{-1} u_{i}(x),
$$

where $x_{\sigma}$ is chosen so that $d\left(x_{\sigma}, x_{i}\right)=\sigma$. Note that

$$
L_{g_{i}} w_{i}+K u_{i}\left(x_{\sigma}\right)^{p_{i}-1} f_{i}^{-\tau_{i}} w_{i}^{p_{i}}=0 .
$$

The Harnack inequality implies that, for every $\epsilon>0$, there exists a constant $C_{\epsilon}>0$ such that

$$
C_{\epsilon}^{-1} \leq w_{i}(x) \leq C_{\epsilon}
$$

if $d(x, \bar{x})>\epsilon$. From Claim 1 we know that $u_{i}\left(x_{\sigma}\right)^{p_{i}-1} \rightarrow 0$ as $i \rightarrow \infty$, and then standard elliptic theory shows that, after maybe passing to a subsequence,

$$
w_{i} \rightarrow w \text { in } C_{l o c}^{2}\left(B_{\sigma}(\bar{x})\right),
$$

and $w$ satisfies

$$
L_{g} w=0, w>0 .
$$

Since the blowup is isolated simple, the function $\hat{u}_{i}(r)$ is decreasing in the interval $\left(R_{i} M_{i}^{-\frac{p_{i}-1}{2}}, \rho\right)$. Taking the limit, we conclude that $\hat{w}(r)$ is decreasing in the whole interval $(0, \rho)$.

As a consequence, $w$ is singular at the origin. 
It follows from the results contained in the appendix in [9] that

$$
-\int_{B_{\eta}\left(x_{i}\right)} \Delta_{g_{i}} w_{i}=-\int_{\partial B_{\eta}\left(x_{i}\right)} \frac{\partial w_{i}}{\partial \nu}=-\int_{\partial B_{\eta}(\bar{x})} \frac{\partial w}{\partial \nu}+o(1)>c>0
$$

for each $i$, where $\eta>0$ is sufficiently small.

On the other hand,

$$
\begin{aligned}
-\int_{B_{\eta}\left(x_{i}\right)} \Delta_{g_{i}} w_{i} & =\int_{B_{\eta}\left(x_{i}\right)}\left[K u_{i}\left(x_{\sigma}\right)^{-1} f_{i}^{-\tau_{i}} u_{i}^{p_{i}}-c(n) R_{g_{i}} w_{i}\right] \\
& \leq K \int_{B_{\eta}\left(x_{i}\right)} u_{i}\left(x_{\sigma}\right)^{-1} f_{i}^{-\tau_{i}} u_{i}^{p_{i}} \leq c u_{i}\left(x_{\sigma}\right)^{-1} M_{i}^{-1} .
\end{aligned}
$$

Here we have used Claim 3. Claim 4 now follows from inequalities (4.23) and (4.24).

Now we are ready to prove Proposition 4.5.

Suppose the inequality (4.9) is not true. Then there exists a sequence $\tilde{x}_{i}$, with $d\left(\tilde{x}_{i}, x_{i}\right) \leq \frac{\rho_{1}}{2}, \rho_{1}$ small, such that

$$
u_{i}\left(\tilde{x}_{i}\right) u_{i}\left(x_{i}\right) d\left(\tilde{x}_{i}, x_{i}\right)^{n-2} \rightarrow \infty
$$

as $i \rightarrow \infty$.

Then, from Proposition 4.3, $R_{i} u_{i}\left(x_{i}\right)^{-\frac{p_{i}-1}{2}} \leq \tilde{r}_{i} \leq \frac{\rho_{1}}{2}$, where $\tilde{r}_{i}=$ $d\left(\tilde{x}_{i}, x_{i}\right)$. Define

$$
\tilde{v}_{i}(y)=\tilde{r}_{i}^{\frac{2}{p_{i}-1}} u_{i}\left(\tilde{r}_{i} y\right), \quad|y|<2 .
$$

Now it is not difficult to see that the origin is an isolated simple blowup point for $\tilde{v}_{i}$, and Claim 4 implies, together with the Harnack inequality,

$$
\max _{|y|=1} \tilde{v}_{i}(0) \tilde{v}_{i}(y) \leq C .
$$

This contradicts the limit in (4.25) and we finish the proof of inequality (4.9).

For the proof of inequality (4.10), recall that the Green function always exists when $\rho_{1}$ is sufficiently small. Now observe that the inequality holds where $d_{g_{i}}\left(x, x_{i}\right)=R_{i} M_{i}^{-\frac{p_{i}-1}{2}}$ because $G_{i}\left(x_{i}, x\right)=O\left(r^{2-n}\right)$ and where $d_{g_{i}}\left(x, x_{i}\right)=\rho_{1}$ because the Green function vanishes in this case and we are dealing with positive functions. Since

$$
L_{g_{i}}\left(u_{i}\left(x_{i}\right) u_{i}\right) \leq 0=L_{g_{i}} G_{i}
$$

we can apply the maximum principle on the region $\left\{x: R_{i} M_{i}^{-\frac{p_{i}-1}{2}} \leq\right.$ $\left.d_{g_{i}}\left(x, x_{i}\right) \leq \rho_{1}\right\}$ to get the desired inequality. 
COROllary 4.6. Under the hypotheses of Proposition 4.5. after maybe passing to a subsequence,

$$
M_{i} u_{i}(x) \rightarrow h \text { in } C_{l o c}^{2}\left(B_{\rho_{1}}(\bar{x}) \backslash\{\bar{x}\}\right),
$$

where $h$ is a positive solution to the linear equation $L_{g}(h)=0$, with a nonremovable singularity at $\bar{x}$. (Here $g$ stands for the limit metric.)

Proof. Observe that the function $M_{i} u_{i}(x)$ satisfies

$$
L_{g_{i}}\left(M_{i} u_{i}(x)\right)+K M_{i}^{1-p_{i}} f_{i}^{-\tau_{i}}(x)\left(M_{i} u_{i}(x)\right)^{p_{i}}=0 .
$$

The previous Proposition implies $M_{i} u_{i}(x)$ is uniformly bounded in compact sets contained in $B_{\rho_{1}}(\bar{x}) \backslash\{\bar{x}\}$, and then standard elliptic estimates show that, after extracting a subsequence, $M_{i} u_{i}(x) \rightarrow h$ in $C_{l o c}^{2}\left(B_{\rho_{1}}(\bar{x}) \backslash\right.$ $\{\bar{x}\})$. Since $M_{i} \rightarrow \infty, L_{g} h=0$.

Because of inequality (4.10), taking the limit, one sees that $h$ is singular. This finishes the proof.

q.e.d.

\section{Symmetry estimates}

In this section we will estimate the difference between solutions to our equation and standard symmetric functions, which will be solutions to the corresponding critical equation in the Euclidean setting.

The next lemma gives us an estimate on $\left|v_{i}-U_{0}\right|$, depending on $M_{i}$ and $\tau_{i}=\frac{n+2}{n-2}-p_{i}$. This is the first step towards the symmetry estimates, not depending on $\tau_{i}$, we will prove later.

LEMMA 5.1. Let $u_{i}$ be a sequence of positive functions satisfying equation (4.1) and $x_{i} \rightarrow \bar{x}$ be an isolated simple blowup point. Then there exists $\delta>0$ such that

$$
\left|v_{i}(y)-U_{0}(y)\right| \leq C\left\{\begin{array}{llr}
\max \left\{M_{i}^{-2}, \tau_{i}\right\} & \text { if } & n=4,5, \\
\max \left\{\left(\log M_{i}\right) M_{i}^{-2}, \tau_{i}\right\} & \text { if } & n=6, \\
\max \left\{M_{i}^{-2} M_{i}^{\frac{2(n-6)}{n-2}}, \tau_{i}\right\} & \text { if } & n \geq 7
\end{array}\right.
$$

for $|y| \leq \delta M_{i}^{\frac{p_{i}-1}{2}}$, where $\tau_{i}=\frac{n+2}{n-2}-p_{i}$.

Proof. Set $l_{i}=\delta M_{i}^{\frac{p_{i}-1}{2}}$, and $\Lambda_{i}=\max _{|y| \leq l_{i}}\left|v_{i}-U_{0}\right|=v_{i}\left(y_{i}\right)-$ $U_{0}\left(y_{i}\right)$, for a certain $\left|y_{i}\right| \leq l_{i}$. 
We observe that if there exists a constant $c>0$ such that $\left|y_{i}\right| \geq c l_{i}$ for every $i$, then inequality $v_{i} \leq c U_{0}$ automatically implies the stronger inequality

$$
\left|\left(v_{i}-U_{0}\right)(y)\right| \leq C M_{i}^{-2}
$$

since

$$
\Lambda_{i}=\left|v_{i}-U_{0}\right|\left(y_{i}\right) \leq C\left|y_{i}\right|^{2-n} \leq C l_{i}^{2-n} \leq C M_{i}^{-2} .
$$

So for large $i$ we will have $\left|y_{i}\right| \leq \frac{l_{i}}{2}$. We are using that $M_{i}^{\tau_{i}} \rightarrow 1$ as $i \rightarrow \infty$.

Define

$$
w_{i}(y)=\Lambda_{i}^{-1}\left(v_{i}(y)-U_{0}(y)\right)
$$

Then $w_{i}$ satisfies

$$
L_{h_{i}} w_{i}+b_{i} w_{i}=Q_{i}(y)
$$

where

$$
b_{i}(y)=K \tilde{f}_{i}^{-\tau_{i}} \frac{v_{i}^{p_{i}}-U_{0}^{p_{i}}}{v_{i}-U_{0}}(y)
$$

and

$$
\begin{aligned}
& Q_{i}(y)=\Lambda_{i}^{-1}\left\{c(n) M_{i}^{-\left(p_{i}-1\right)} R_{g_{i}}\left(M_{i}^{-\frac{p_{i}-1}{2}} y\right) U_{0}(y)\right. \\
& \left.+M_{i}^{-(1+N) \frac{p_{i}-1}{2}} O\left(|y|^{N}\right)|y|\left(1+|y|^{2}\right)^{-\frac{n}{2}}+K\left(U_{0}^{\frac{n+2}{n-2}}-\tilde{f}_{i}^{-\tau_{i}} U_{0}^{p_{i}}\right)\right\}
\end{aligned}
$$

where $\tilde{f}_{i}(y)=f_{i}\left(M_{i}^{-\frac{p_{i}-1}{2}} y\right),\left(h_{i}\right)_{k l}(y)=\left(g_{i}\right)_{k l}\left(M_{i}^{-\frac{p_{i}-1}{2}} y\right)$ and $O\left(|y|^{N}\right)$ comes from the expansion of the volume element in conformal normal coordinates and $N$ is as big as we want.

Since the blowup is isolated simple, from inequality $v_{i} \leq c U_{0}$, it is easy to check, for example,

$$
b_{i}(y) \leq c(1+|y|)^{-3}
$$

for $|y| \leq l_{i}$.

We will choose $\delta$ small enough to guarantee the existence of the Green's function for the conformal Laplacian on a ball of radius $\delta$, with respect to a Dirichlet boundary condition.

The Green's representation formula gives

$w_{i}(y)=\int_{B_{i}} G_{i, L}(y, \eta)\left(b_{i}(\eta) w_{i}(\eta)-Q_{i}(\eta)\right) d \eta-\int_{\partial B_{i}} \frac{\partial G_{i, L}}{\partial \nu}(y, \eta) w_{i}(\eta) d s$

where $B_{i}$ stands for $B_{l_{i}}(0)$ and $G_{i, L}$ is the Green function of $L_{h_{i}}$ in $B_{i}$.

We will need the following lemma proved in [3] : 
Lemma 5.2. Suppose $w$ is a solution to the equation

$$
\Delta w+n(n+2) U_{0}^{\frac{4}{n-2}} w=0 \text { in } \mathbb{R}^{n} .
$$

If $\lim _{|y| \rightarrow \infty} w(y)=0$, then there exist constants $c_{0}, c_{1}, \ldots, c_{n}$ such that

$$
w(y)=c_{0}\left(\frac{n-2}{2} U_{0}+y \cdot \nabla U_{0}\right)+\sum_{j=1}^{n} c_{j} \frac{\partial U_{0}}{\partial y_{j}} .
$$

Remark: The functions $\frac{n-2}{2} U_{0}+y \cdot \nabla U_{0}$ and $\frac{\partial U_{0}}{\partial y_{j}}, j=1, \ldots, n$, are solutions to the equation (5.4).

The proof of the Lemma 5.1 is by contradiction. Set

$$
t_{i}=\left\{\begin{array}{llr}
M_{i}^{-2} & \text { if } & n=4,5, \\
\left(\log M_{i}\right) M_{i}^{-2} & \text { if } & n=6, \\
M_{i}^{-2} M_{i}^{\frac{2(n-6)}{n-2}} & \text { if } & n \geq 7 .
\end{array}\right.
$$

If the proposition is false, we necessarily have

$$
\Lambda_{i}^{-1} \max \left\{t_{i}, \tau_{i}\right\} \rightarrow 0
$$

as $i \rightarrow \infty$, which implies that

$$
\Lambda_{i}^{-1} t_{i} \rightarrow 0, \quad \Lambda_{i}^{-1} \tau_{i} \rightarrow 0 .
$$

Since $R=O\left(r^{2}\right)$ in conformal normal coordinates, we can get the following estimate:

$$
\begin{aligned}
\left|Q_{i}(y)\right| & \leq c \Lambda_{i}^{-1}\left\{M_{i}^{-\frac{8}{n-2}}|y|^{2}(1+|y|)^{2-n}\right. \\
& +M_{i}^{-(1+N) \frac{p_{i}-1}{2}} O\left(|y|^{N}\right)|y|\left(1+|y|^{2}\right)^{-\frac{n}{2}} \\
& \left.+\tau_{i}\left(\left|\log U_{0}\right|+\left|\log \tilde{f}_{i}\right|\right)(1+|y|)^{-n-2}\right\} .
\end{aligned}
$$

Using the estimates (5.2) and (5.6), we get from the Green's representation formula (5.3) that $w_{i}$ is bounded in $C_{l o c}^{2}$, and

$$
\left|w_{i}(y)\right| \leq c\left[(1+|y|)^{-1}+c \Lambda_{i}^{-1} t_{i}\right]
$$

for $|y| \leq \frac{\delta}{2} M_{i}^{\frac{p_{i}-1}{2}}$. We are using that $\left|w_{i}(y)\right| \leq C \Lambda_{i}^{-1} M_{i}^{-2}$ when $|y|=$ $\frac{\delta}{2} M_{i}^{\frac{p_{i}-1}{2}}$, and also that $\left|G_{i, L}(y, \eta)\right| \leq C|y-\eta|^{2-n}$ for $|y| \leq \frac{l_{i}}{2}$. 
Then, by standard elliptic estimates, there exists a subsequence, also denoted $w_{i}$, converging to $w$ satisfying

$$
\left\{\begin{array}{r}
\Delta w+n(n+2) U_{0}^{\frac{4}{n-2}}(y) w=0 \text { in } \mathbb{R}^{n}, \\
|w(y)| \leq c(1+|y|)^{-1} .
\end{array}\right.
$$

So, the Lemma 5.2 implies that

$$
w(y)=c_{0}\left(\frac{n-2}{2} U_{0}+y \cdot \nabla U_{0}\right)+\sum_{j=1}^{n} c_{j} \frac{\partial U_{0}}{\partial y_{j}} .
$$

The conditions $w(0)=\frac{\partial w}{\partial y_{j}}(0)=0$ show that $c_{j}=0$ for every $j$, in other words, $w(y) \equiv 0$. From here we conclude that $\left|y_{i}\right| \rightarrow \infty$ as $i \rightarrow \infty$.

This contradicts the estimate (5.7) since $w_{i}\left(y_{i}\right)=1$ and $\Lambda_{i}^{-1} t_{i} \rightarrow 0$, and this finishes the proof.

q.e.d.

In the next lemma, we estimate $\tau_{i}$. This result and the Lemma 5.1 give us an estimate on $\left|v_{i}-U_{0}\right|$ independent of $\tau_{i}$.

LEMma 5.3. Under the same hypotheses in Lemma 5.1,

$$
\tau_{i} \leq C\left\{\begin{array}{llr}
M_{i}^{-2} & \text { if } & n=4,5, \\
\left(\log M_{i}\right) M_{i}^{-2} & \text { if } & n=6, \\
M_{i}^{-2} M_{i}^{\frac{2(n-6)}{n-2}} & \text { if } & n \geq 7 .
\end{array}\right.
$$

Proof. The proof will be again by contradiction and recall the definition (5.5). If the lemma is not true, then Lemma 5.1] implies that

$$
\left|v_{i}(y)-U_{0}(y)\right| \leq C \tau_{i}
$$

Define

$$
w_{i}(y)=\tau_{i}^{-1}\left(v_{i}-U_{0}\right)(y)
$$

so $w_{i}$ is uniformly bounded. The equation satisfied by $w_{i}$ is

$$
L_{h_{i}} w_{i}+b_{i} w_{i}=\tilde{Q}_{i}(y)
$$

where

$$
b_{i}(y)=K \tilde{f}_{i}^{-\tau_{i}} \frac{v_{i}^{p_{i}}-U_{0}^{p_{i}}}{v_{i}-U_{0}}(y)
$$


and

$$
\begin{aligned}
\tilde{Q}_{i}(y) & =\tau_{i}^{-1}\left\{c(n) M_{i}^{-\left(p_{i}-1\right)} R_{g_{i}}\left(M_{i}^{-\frac{p-1}{2}} y\right) U_{0}(y)\right. \\
& +M_{i}^{-(1+N) \frac{p_{i}-1}{2}} O\left(|y|^{N}\right)|y|\left(1+|y|^{2}\right)^{-\frac{n}{2}} \\
& \left.+K\left(U_{0}^{\frac{n+2}{n-2}}-\tilde{f}_{i}^{-\tau_{i}} U_{0}^{p_{i}}\right)\right\} .
\end{aligned}
$$

If the lemma is not true, then $\tau_{i}^{-1} t_{i} \rightarrow 0$ as $i \rightarrow \infty$.

We have

$$
\begin{aligned}
\left|\tilde{Q}_{i}(y)\right| & \leq c \tau_{i}^{-1}\left\{M_{i}^{-\frac{8}{n-2}}|y|^{2}(1+|y|)^{2-n}\right. \\
& +M_{i}^{-(1+N) \frac{p_{i}-1}{2}} O\left(|y|^{N}\right)|y|\left(1+|y|^{2}\right)^{-\frac{n}{2}} \\
& \left.+\tau_{i}\left(\left|\log U_{0}\right|+\left|\log \tilde{f}_{i}\right|\right)(1+|y|)^{-n-2}\right\} .
\end{aligned}
$$

By elliptic linear theory we can suppose $w_{i} \rightarrow w$ in compact subsets. If $\psi(y)=\frac{n-2}{2} U_{0}(y)+y \cdot \nabla U_{0}(y)$, then,

$$
\begin{aligned}
& \int_{|y| \leq \frac{l_{i}}{2}} \psi(y) \tau_{i}^{-1}\left(M_{i}^{-\frac{8}{n-2}}|y|^{2}(1+|y|)^{2-n}\right. \\
& \left.\quad+M_{i}^{-(1+N) \frac{p_{i}-1}{2}} O\left(|y|^{N}\right)|y|\left(1+|y|^{2}\right)^{-\frac{n}{2}}\right) \rightarrow 0 .
\end{aligned}
$$

Note that when $i \rightarrow \infty$ we have:

$$
\tau_{i}^{-1} K\left(U_{0}^{\frac{n+2}{n-2}}-\tilde{f}_{i}^{-\tau_{i}} U_{0}^{p_{i}}\right) \rightarrow K\left(\log U_{0}(y)+\log f(\bar{x})\right) U_{0}^{\frac{n+2}{n-2}}
$$

pointwise. It is not difficult to check that

$$
\int_{\mathbb{R}^{n}} \psi(y) U_{0}^{\frac{n+2}{n-2}}(y) d y=0 .
$$

Therefore we can conclude

$$
\lim _{i \rightarrow \infty} \int_{|y| \leq \frac{l_{i}}{2}} \psi(y) \tilde{Q}_{i}(y) d y=n(n-2) \int_{\mathbb{R}^{n}} \psi(y)\left(\log U_{0}(y)\right) U_{0}^{\frac{n+2}{n-2}}(y) d y .
$$

On the other hand, integration by parts shows that

$$
\begin{aligned}
& \int_{|y| \leq \frac{l_{i}}{2}} \psi(y) \tilde{Q}_{i}(y) d y=\int_{|y| \leq \frac{l_{i}}{2}} \psi(y)\left(L_{h_{i}} w_{i}+b_{i} w_{i}\right) d y \\
& =\int_{|y| \leq \frac{l_{i}}{2}}\left(L_{h_{i}} \psi(y)+b_{i} \psi\right) w_{i} d y+\int_{|y|=\frac{l_{i}}{2}}\left(\psi \frac{\partial w_{i}}{\partial r}-w_{i} \frac{\partial \psi}{\partial r}\right) d \sigma
\end{aligned}
$$


The integral on the boundary goes to zero when $i \rightarrow \infty$ because

$$
\left\{\begin{array}{c}
|\psi|=O\left(r^{2-n}\right),|\nabla \psi|=O\left(r^{1-n}\right) \\
\left|w_{i}\left(\frac{l_{i}}{2}\right)\right| \leq c \tau_{i}^{-1} M_{i}^{-2},\left|\nabla w_{i}\left(\frac{l_{i}}{2}\right)\right| \leq c \tau_{i}^{-1} M_{i}^{-2} l_{i}^{-1} .
\end{array}\right.
$$

Taking the limit when $i \rightarrow \infty$, we would have

$$
\lim _{i \rightarrow \infty} \int_{|y| \leq \frac{l_{i}}{2}} \psi(y) \tilde{Q}_{i}(y) d y=\int_{\mathbb{R}^{n}}\left(\Delta \psi(y)+n(n+2) U_{0}^{\frac{4}{n-2}} \psi\right) w d y=0
$$

because $\Delta \psi(y)+n(n+2) U_{0}^{\frac{4}{n-2}} \psi=0$.

This is a contradiction because

$$
n(n-2) \int_{\mathbb{R}^{n}} \psi(y)\left(\log U_{0}(y)\right) U_{0}^{\frac{n+2}{n-2}}(y) d y>0 .
$$

To see this, first note that

$$
\psi(y)=\frac{n-2}{2} \frac{1-r^{2}}{\left(1+r^{2}\right)^{\frac{n}{2}}} .
$$

Observe that

$$
\begin{aligned}
& \int_{\mathbb{R}^{n}} \psi(y)\left(\log U_{0}(y)\right) U_{0}^{\frac{n+2}{n-2}}(y) d y= \\
& -\frac{(n-2)^{2}}{4} \sigma_{n-1} \int_{0}^{\infty} \frac{1-r^{2}}{\left(1+r^{2}\right)^{n+1}} r^{n-1} \log \left(1+r^{2}\right) d r
\end{aligned}
$$

and after changing variables $r=s^{-1}$, we get

$$
\int_{0}^{\infty} \frac{1-r^{2}}{\left(1+r^{2}\right)^{n+1}} r^{n-1} \log \left(1+r^{2}\right) d r=2 \int_{1}^{\infty} \frac{1-r^{2}}{\left(1+r^{2}\right)^{n+1}} r^{n-1} \log r d r .
$$

Now inequality (5.12) follows immediately, and that finishes the proof of the lemma.

q.e.d.

The Lemmas 5.1 and 5.3 together imply our symmetry estimate:

Proposition 5.4. Let $u_{i}$ be a sequence of positive functions satisfying equation (4.1) and $x_{i} \rightarrow \bar{x}$ be an isolated simple blowup point. Then there exists $\delta>0$ such that

$$
\left|v_{i}(y)-U_{0}(y)\right| \leq C\left\{\begin{array}{llr}
M_{i}^{-2} & \text { if } & n=4 \text { or } 5, \\
\left(\log M_{i}\right) M_{i}^{-2} & \text { if } & n=6, \\
M_{i}^{-2} M_{i}^{\frac{2(n-6)}{n-2}} & \text { if } & n \geq 7
\end{array}\right.
$$

for $|y| \leq \delta M_{i}^{\frac{p_{i}-1}{2}}$. 
When $n \geq 6$, by applying the same technique we can also get:

Proposition 5.5. Let $u_{i}$ be a sequence of positive functions satisfying equation (4.1) and $x_{i} \rightarrow \bar{x}$ be an isolated simple blowup point. Then

$$
\left|v_{i}(y)-U_{0}(y)\right| \leq C \begin{cases}M_{i}^{-2} M_{i}^{\frac{2}{n-2}}(1+|y|)^{-1} & \text { if } n=6 \\ M_{i}^{-2} M_{i}^{\frac{2(n-6)}{n-2}}(1+|y|)^{6-n} & \text { if } n \geq 7\end{cases}
$$

Proof. Set

$$
A_{i}= \begin{cases}M_{i}^{-2} M_{i}^{\frac{2}{n-2}} & \text { if } n=6 \\ M_{i}^{-2} M_{i}^{\frac{2(n-6)}{n-2}} & \text { if } n \geq 7\end{cases}
$$

and define

$$
w_{i}(y)=A_{i}^{-1}\left(v_{i}-U_{0}\right)(y)
$$

for $|y| \leq \delta M_{i}^{\frac{p_{i}-1}{2}}$. Then our previous proposition implies $w_{i}$ is uniformly bounded. The equation satisfied is

$$
L_{h_{i}} w_{i}+b_{i} w_{i}=\tilde{Q}_{i}(y)
$$

where

and

$$
b_{i}(y)=K \tilde{f}_{i}^{-} \tau_{i} \frac{v_{i}^{p_{i}}-U_{0}^{p_{i}}}{v_{i}-U_{0}}(y)
$$

$$
\begin{aligned}
& \tilde{Q}_{i}(y)=A_{i}^{-1}\left\{c(n) M_{i}^{-(p-1)} R_{g}\left(M_{i}^{-\frac{p-1}{2}} y\right) U_{0}(y)\right. \\
& \left.+M_{i}^{-(1+N) \frac{p_{i}-1}{2}} O\left(|y|^{N}\right)|y|\left(1+|y|^{2}\right)^{-\frac{n}{2}}+K\left(U_{0}^{\frac{n+2}{n-2}}-\tilde{f}_{i}^{-\tau_{i}} U_{0}^{p_{i}}\right)\right\}
\end{aligned}
$$

Then

The Green's representation formula says that

$w_{i}(y)=\int_{B_{i}} G_{i, L}(y, \eta)\left(b_{i}(\eta) w_{i}(\eta)-\tilde{Q}_{i}(\eta)\right) d \eta-\int_{\partial B_{i}} \frac{\partial G_{i, L}}{\partial \nu}(y, \eta) w_{i}(\eta) d s$,

where $B_{i}$ stands for $B_{l_{i}}(0)$ and $G_{i, L}$ is the Green function of $L_{h_{i}}$ in $B_{i}$. 
Since $\left|G_{i, L}(y, \eta)\right| \leq C|y-\eta|^{2-n}$, for $|y| \leq \frac{l_{i}}{2}$, we get

$$
\left|w_{i}(y)\right| \leq c\left\{(1+|y|)^{-1}+c M_{i}^{-2} A_{i}^{-1}\right\} \leq c(1+|y|)^{-1} .
$$

If $n=6$ or 7 the result follows multiplying the inequality (5.20) by $A_{i}$. If $n \geq 8$ we plug the estimate (5.20) in the representation formula (5.19) until we reach

$$
\left|w_{i}(y)\right| \leq c(1+|y|)^{6-n} .
$$

Multiplying by $A_{i}$ we get the result.

q.e.d.

Remark 1: Once we have estimates (5.14), (5.15) on $v_{i}-U_{0}$, we can also get:

$$
\left|\nabla\left(v_{i}-U_{0}\right)(y)\right| \leq C\left\{\begin{array}{llr}
M_{i}^{-2}(1+|y|)^{-1} & \text { if } & n=4 \text { or } 5 \\
M_{i}^{-2} M_{i}^{\frac{2}{n-2}}(1+|y|)^{-2} & \text { if } & n=6 \\
M_{i}^{-2} M_{i}^{\frac{2(n-6)}{n-2}}(1+|y|)^{5-n} & \text { if } & n \geq 7
\end{array}\right.
$$

and

$$
\left|\nabla^{2}\left(v_{i}-U_{0}\right)(y)\right| \leq C\left\{\begin{array}{llr}
M_{i}^{-2}(1+|y|)^{-2} & \text { if } & n=4 \text { or } 5 \\
M_{i}^{-2} M_{i}^{\frac{2}{n-2}}(1+|y|)^{-3} & \text { if } & n=6 \\
M_{i}^{-2} M_{i}^{\frac{2(n-6)}{n-2}}(1+|y|)^{4-n} & \text { if } & n \geq 7 .
\end{array}\right.
$$

Remark 2: If $h$ is as in the Corollary 4.6, the estimates (5.14) and (5.15) imply that:

$$
\left.|h(x)-| x\right|^{2-n} \mid \leq C\left\{\begin{array}{llr}
1 & \text { if } & n=4,5 \\
|x|^{-1} & \text { if } & n=6 \\
|x|^{6-n} & \text { if } & n \geq 7 .
\end{array}\right.
$$

Since that gives the asymptotic behavior of the Green function of the conformal Laplacian in conformal normal coordinates (see [7]), in some sense our symmetry estimates cannot be improved.

\section{Local blowup analysis}

Now let us turn our attention to the applications of these symmetry estimates.

In the first application, we will show that the Weyl tensor of the metric has to vanish at an isolated simple blowup point, when $n \geq 6$. This result had been proposed by R. Schoen ([14]). This will allow us to 
use the Positive Mass Theorem in the proof of compactness of solutions to the Yamabe problem, when the dimension is 6 or 7 .

THEOREM 6.1. Let $u_{i}$ be a sequence of positive functions satisfying equation (4.1) and $x_{i} \rightarrow \bar{x}$ be an isolated simple blowup point. If $n \geq 6$, then

$$
W_{g}(\bar{x})=0 .
$$

Proof. We will use the Pohozaev identity (2.8) to $u_{i}$ in a ball of radius $r$ :

$$
\begin{aligned}
& P\left(r, u_{i}\right)= \\
& -\int_{|x| \leq r}\left(x^{m} \partial_{m} u_{i}+\frac{n-2}{2} u_{i}\right)\left(\left(g^{k l}-\delta^{k l}\right) \partial_{k l} u_{i}+\partial_{k} g^{k l} \partial_{l} u_{i}\right) d x \\
& -c(n) \int_{|x| \leq r}\left(\frac{1}{2} x^{k} \partial_{k} R+R\right) u_{i}^{2} d x+c(n) \frac{r}{2} \int_{|x|=r} R u_{i}^{2} d \sigma_{r} \\
& \quad+\left(\frac{n}{p_{i}+1}-\frac{n-2}{2}\right) \int_{|x| \leq r} K f_{i}^{-\tau_{i}} u_{i}^{p_{i}+1} d x \\
& \quad-\frac{\tau_{i}}{p_{i}+1} \int_{|x| \leq r} K f_{i}^{-\tau_{i}-1}\left(x^{m} \partial_{m} f_{i}\right) u_{i}^{p_{i}+1} d x .
\end{aligned}
$$

Using conformal normal coordinates we can get rid of the terms involving $|g|$.

Recall

$$
\int_{|x|=r}\left(\frac{n-2}{2} u_{i} \frac{\partial u_{i}}{\partial r}-\frac{r}{2}\left|\nabla u_{i}\right|^{2}+r\left|\frac{\partial u_{i}}{\partial r}\right|^{2}+\frac{1}{p_{i}+1} K f_{i}^{-\tau_{i}} r u_{i}^{p_{i}+1}\right) d \sigma_{r} .
$$

Since we have that $M_{i} u_{i} \rightarrow h$ in the $C^{2}$ topology on compact subsets of $\left.B_{\rho}(\bar{x}) \backslash\{\bar{x}\}\right)$, we conclude

$$
M_{i}^{2}\left|P\left(r, u_{i}\right)\right| \leq c<\infty .
$$

The same holds for

$$
M_{i}^{2}\left|\int_{|x|=r} R u_{i}^{2} d \sigma(r)\right| \leq c<\infty .
$$


The change of variables $y=M_{i}^{\frac{p_{i}-1}{2}} x$ yields

$$
\begin{aligned}
& A_{i}(r):= \\
& M_{i}^{2}\left\{-\int_{|x| \leq r}\left(x^{m} \partial_{m} u_{i}+\frac{n-2}{2} u_{i}\right)\left(\left(g^{k l}-\delta^{k l}\right) \partial_{k l} u_{i}+\partial_{k} g^{k l} \partial_{l} u_{i}\right) d x\right. \\
& \left.-c(n) \int_{|x| \leq r}\left(\frac{1}{2} x^{k} \partial_{k} R+R\right) u_{i}^{2} d x\right\} \\
& =-M_{i}^{2} M_{i}^{2+(2-n) \frac{p_{i}-1}{2}} \int_{|y| \leq r M_{i}^{\frac{p_{i}-1}{2}}\left\{\left(y^{m} \partial_{m} v_{i}+\frac{n-2}{2} v_{i}\right)\right.} \\
& \left(\left(g^{k l}-\delta^{k l}\right)\left(M_{i}^{-\frac{p_{i}-1}{2}} y\right) \partial_{k l} v_{i}+M_{i}^{-\frac{p_{i}-1}{2}} \partial_{k} g^{k l}\left(M_{i}^{-\frac{p_{i}-1}{2}} y\right) \partial_{l} v_{i}\right) \\
& \left.+c(n) M_{i}^{-\left(p_{i}-1\right)}\left(\frac{1}{2} y^{k} \partial_{k} R\left(M_{i}^{-\frac{p_{i}-1}{2}} y\right)+R\left(M_{i}^{-\frac{p_{i}-1}{2}} y\right)\right) v_{i}^{2}\right\} d y .
\end{aligned}
$$

Note that $M_{i}^{2+(2-n) \frac{p_{i}-1}{2}} \rightarrow 1$. Define

$$
\begin{aligned}
& \hat{A}_{i}(r)= \\
& -M_{i}^{2} M_{i}^{2+(2-n) \frac{p_{i}-1}{2}} \int_{|y| \leq r M_{i}^{\frac{p_{i}-1}{2}}}\left\{\left(y^{m} \partial_{m} U_{0}+\frac{n-2}{2} U_{0}\right)\right. \\
& \left(\left(g^{k l}-\delta^{k l}\right)\left(M_{i}^{-\frac{p_{i}-1}{2}} y\right) \partial_{k l} U_{0}+M_{i}^{-\frac{p_{i}-1}{2}} \partial_{k} g^{k l}\left(M_{i}^{-\frac{p_{i}-1}{2}} y\right) \partial_{l} U_{0}\right) \\
& \left.+c(n) M_{i}^{-\left(p_{i}-1\right)}\left(\frac{1}{2} y^{k} \partial_{k} R\left(M_{i}^{-\frac{p_{i}-1}{2}} y\right)+R\left(M_{i}^{-\frac{p_{i}-1}{2}} y\right)\right) U_{0}^{2}\right\} d y .
\end{aligned}
$$

Then one can check

$$
\begin{aligned}
& \quad\left|A_{i}(r)-\hat{A}_{i}(r)\right| \\
& \quad \leq c M_{i}^{2} M_{i}^{-\frac{4}{n-2}} \int_{|y| \leq r M_{i}^{\frac{p_{i}-1}{2}}}\left\{\left|v_{i}-U_{0}\right|(y)(1+|y|)^{2-n}\right. \\
& \left.+\left|\nabla\left(v_{i}-U_{0}\right)\right|(1+|y|)^{3-n}+\left|\nabla^{2}\left(v_{i}-U_{0}\right)\right|(1+|y|)^{4-n}\right\} d y .
\end{aligned}
$$

If $n=6$, then

$$
\left|A_{i}(r)-\hat{A}_{i}(r)\right| \leq c M_{i}^{-\frac{2}{n-2}} \int_{|y| \leq r M_{i}^{\frac{p_{i}-1}{2}}}(1+|y|)^{1-n} d y .
$$

When $n \geq 7$,

$$
\left|A_{i}(r)-\hat{A}_{i}(r)\right| \leq c M_{i}^{-\frac{4}{n-2}} M_{i}^{(n-6) \frac{2}{n-2}} \int_{|y| \leq r M_{i}^{\frac{p_{i}-1}{2}}}(1+|y|)^{8-2 n} d y .
$$


Then

$$
\left|A_{i}(r)-\hat{A}_{i}(r)\right| \leq\left\{\begin{array}{llr}
C & \text { if } & n=6,7 \\
C\left(\log M_{i}\right) & \text { if } & n=8 \\
C M_{i}^{(n-8) \frac{2}{n-2}} & \text { if } & n \geq 9 .
\end{array}\right.
$$

Also observe that if we choose $r$ sufficiently small,

$$
\begin{aligned}
& \left(\frac{n}{p_{i}+1}-\frac{n-2}{2}\right) \int_{|x| \leq r} K u_{i}^{p_{i}+1} d x \\
- & \frac{\tau_{i}}{p_{i}+1} \int_{|x| \leq r} K f_{i}^{-\tau_{i}-1}\left(x^{m} \partial_{m} f_{i}\right) u_{i}^{p_{i}+1} d x \geq 0,
\end{aligned}
$$

so we obtain, from identity (6.1), estimates (6.3), (6.4), (6.7) and inequality (6.8) that

$$
\hat{A}_{i}(r) \leq\left\{\begin{array}{llr}
C & \text { if } & n=6,7 \\
C\left(\log M_{i}\right) & \text { if } & n=8 \\
C M_{i}^{(n-8) \frac{2}{n-2}} & \text { if } & n \geq 9 .
\end{array}\right.
$$

We will need the Taylor series, for each $i$,

$$
\begin{gathered}
R(x)=p_{2}(x)+p_{3}(x)+e(x) \\
\left(R+\frac{1}{2} x^{k} \partial_{k} R\right)(x)=2 p_{2}(x)+\frac{5}{2} p_{3}(x)+e^{\prime}(x)
\end{gathered}
$$

where $p_{i}$ is a homogeneous polynomial of degree $i$ and $|e(x)|,\left|e^{\prime}(x)\right| \leq$ $c|x|^{4}$.

Let us denote by

$$
\tilde{u}_{i}(x)=M_{i}^{\frac{n-2}{4} \tau_{i}}\left(\frac{M_{i}^{-\frac{p_{i}-1}{2}}}{M_{i}^{-\left(p_{i}-1\right)}+|x|^{2}}\right)^{\frac{n-2}{2}}
$$

and it is not difficult to see that, changing variables, estimates on $\mid v_{i}-$ $U_{0} \mid$ yield estimates on $\left|u_{i}-\tilde{u}_{i}\right|$.

Note that

$$
M_{i}^{2} \int_{|x| \leq r}\left(\frac{1}{2} x^{k} \partial_{k} e+e\right) \tilde{u}_{i}^{2} d x \leq\left\{\begin{array}{llr}
C & \text { if } & n=6,7 \\
C\left(\log M_{i}\right) & \text { if } & n=8 \\
C M_{i}^{(n-8) \frac{2}{n-2}} & \text { if } & n \geq 9
\end{array}\right.
$$


Then, using symmetry of $\tilde{u}_{i}$

$$
-c(n) \int_{|x| \leq r} 2 p_{2}(x)\left(M_{i} \tilde{u}_{i}\right)^{2} d x \leq c\left\{\begin{array}{llr}
C & \text { if } & n=6,7 \\
C\left(\log M_{i}\right) & \text { if } & n=8 \\
C M_{i}^{(n-8) \frac{2}{n-2}} & \text { if } & n \geq 9
\end{array}\right.
$$

But, on the other hand, since $\Delta R(0)=-\frac{1}{6}|W(0)|^{2}$ in conformal normal coordinates, we obtain

$$
\begin{aligned}
& -c(n) M_{i}^{2} \int_{|x| \leq r} 2 p_{2}(x) \tilde{u}_{i}^{2} d x \geq \\
& \qquad\left\{\begin{array}{lll}
C\left|W\left(x_{i}\right)\right|^{2} M_{i}^{2} M_{i}^{-4 \frac{2}{n-2}}\left(\log M_{i}\right) & \text { if } & n=6, \\
C\left|W\left(x_{i}\right)\right|^{2} M_{i}^{2} M_{i}^{-4 \frac{2}{n-2}} & \text { if } & n \geq 7 .
\end{array}\right.
\end{aligned}
$$

So

$$
\left|W_{g_{i}}\left(x_{i}\right)\right|^{2} \leq\left\{\begin{array}{llc}
c\left(\log M_{i}\right)^{-1} & \text { if } & n=6 \\
c M_{i}^{-\frac{2}{n-2}} & \text { if } & n=7 \\
c M_{i}^{-\frac{4}{n-2}}\left(\log M_{i}\right) & \text { if } & n=8 \\
c M_{i}^{-\frac{4}{n-2}} & \text { if } & n \geq 9
\end{array}\right.
$$

And taking the limit we are done.

q.e.d.

The next result concerns the local asymptotic analysis at a blowup point. It will be used together with the Positive Mass Theorem to exclude the possibility of blowup phenomenon on manifolds not conformally diffeomorphic to the sphere.

Define

$$
P^{\prime}(r, v)=\int_{|x|=r}\left(\frac{n-2}{2} v \frac{\partial v}{\partial \nu}-\frac{r}{2}|\nabla v|^{2}+r\left|\frac{\partial v}{\partial \nu}\right|^{2}\right) d \sigma(r) .
$$

THEOREM 6.2. Let $u_{i}$ be a sequence of positive functions satisfying equation (4.1) and $x_{i} \rightarrow \bar{x}$ be an isolated simple blowup point. and suppose $4 \leq n \leq 7$. If $u_{i}\left(x_{i}\right) u_{i} \rightarrow h$ away from the origin, then

$$
\liminf _{r \rightarrow 0} P^{\prime}(r, h) \geq 0
$$


Proof. That is another application of the Pohozaev identity (2.7) and the symmetry estimates:

$$
\begin{aligned}
& P\left(r, u_{i}\right)=-\int_{|x| \leq r}\left(x^{m} \partial_{m} u_{i}+\frac{n-2}{2} u_{i}\right) \\
& \quad\left(\left(g^{k l}-\delta^{k l}\right) \partial_{k l} u_{i}+\partial_{k} g^{k l} \partial_{l} u_{i}-c(n) R u_{i}\right) d x \\
& +\left(\frac{n}{p_{i}+1}-\frac{n-2}{2}\right) \int_{|x| \leq r} K f_{i}^{-\tau_{i}} u_{i}^{p_{i}+1} d x \\
& -\frac{\tau_{i}}{p_{i}+1} \int_{|x| \leq r} K f_{i}^{-\tau_{i}-1}\left(x^{m} \partial_{m} f_{i}\right) u_{i}^{p_{i}+1} d x .
\end{aligned}
$$

Firstly observe that

$$
M_{i}^{2} P\left(r, u_{i}\right) \rightarrow P^{\prime}(r, h)
$$

as $i \rightarrow \infty$.

Secondly, as in the previous result, if $r$ is sufficiently small,

$$
\begin{aligned}
& \left(\frac{n}{p_{i}+1}-\frac{n-2}{2}\right) \int_{|x| \leq r} K f_{i}^{-\tau_{i}} u_{i}^{p_{i}+1} d x \\
& -\frac{\tau_{i}}{p_{i}+1} \int_{|x| \leq r} K f_{i}^{-\tau_{i}-1}\left(x^{m} \partial_{m} f_{i}\right) u_{i}^{p_{i}+1} d x \geq 0 .
\end{aligned}
$$

If

$$
\begin{aligned}
& A_{i}(r):=M_{i}^{2}\left\{-\int_{|x| \leq r}\left(x^{m} \partial_{m} u_{i}+\frac{n-2}{2} u_{i}\right)\left(\left(g^{k l}-\delta^{k l}\right) \partial_{k l} u_{i}\right.\right. \\
& \left.\left.\quad+\partial_{k} g^{k l} \partial_{l} u_{i}-c(n) R u_{i}\right) d x\right\}
\end{aligned}
$$

and

$$
\begin{aligned}
& \hat{A}_{i}(r):=M_{i}^{2}\left\{-\int_{|x| \leq r}\left(x^{m} \partial_{m} \tilde{u}_{i}+\frac{n-2}{2} \tilde{u}_{i}\right)\left(\left(g^{k l}-\delta^{k l}\right) \partial_{k l} \tilde{u}_{i}\right.\right. \\
& \left.\left.\quad+\partial_{k} g^{k l} \partial_{l} \tilde{u}_{i}-c(n) R \tilde{u}_{i}\right) d x\right\}
\end{aligned}
$$

then one can check

$$
\left|A_{i}(r)-\hat{A}_{i}(r)\right| \leq\left\{\begin{array}{lll}
c r^{2} & \text { if } & n=4,5 \\
c r & \text { if } & n=6,7
\end{array}\right.
$$

So

$$
\begin{aligned}
& \liminf _{r \rightarrow 0} P^{\prime}(r, h) \geq \\
& c(n) M_{i}^{2} \liminf _{r \rightarrow 0} \int_{|x| \leq r}\left(x^{m} \partial_{m} \tilde{u}_{i}+\frac{n-2}{2} \tilde{u}_{i}\right) R \tilde{u}_{i} d x .
\end{aligned}
$$


If $n=4$ or 5 , since $R=O\left(r^{2}\right)$, this automatically gives

$$
\liminf _{r \rightarrow 0} P^{\prime}(r, h) \geq 0 \text {. }
$$

If $n=6$ or 7 , the fact that $R=O\left(r^{2}\right)$ tells us that we only need to consider the second order term in the expansion of $R$. We are using the symmetry of $\tilde{u}_{i}$ to get rid of the third order term.

The change of variables $y=M_{i}^{\frac{p_{i}-1}{2}} x$ implies that

$$
\begin{aligned}
& M_{i}^{2} \int_{|x| \leq r}\left(x^{m} \partial_{m} \tilde{u}_{i}+\frac{n-2}{2} \tilde{u}_{i}\right) R_{, a b} x^{a} x^{b} \tilde{u}_{i} d x \\
& =(1+o(1)) M_{i}^{\frac{2(n-6)}{n-2}} \int_{|y| \leq r M_{i}^{\frac{p_{i}-1}{2}}}\left(y^{m} \partial_{m} U_{0}+\frac{n-2}{2} U_{0}\right) R_{, a b} y^{a} y^{b} U_{0} d y .
\end{aligned}
$$

But

$$
\begin{aligned}
& \int_{|y| \leq r M_{i}^{\frac{p_{i}-1}{2}}}\left(y^{m} \partial_{m} U_{0}+\frac{n-2}{2} U_{0}\right) R_{, a b} y^{a} y^{b} U_{0} d y \\
& =\int_{0}^{r M_{i}^{\frac{p_{i}-1}{2}}}\left(r \partial_{r} U_{0}+\frac{n-2}{2} U_{0}\right) U_{0} r^{n+1} \frac{\Delta R}{n} \sigma_{n-1} d r \\
& =-\frac{1}{6 n} \sigma_{n-1}|W(0)|^{2} \int_{0}^{r M_{i}^{\frac{p_{i}-1}{2}}}\left(r \partial_{r} U_{0}+\frac{n-2}{2} U_{0}\right) U_{0} r^{n+1} d r \\
& =-\frac{1}{6 n} \sigma_{n-1}|W(0)|^{2}\left(-\frac{n-2}{2} \int_{0}^{r M_{i}^{\frac{p_{i}-1}{2}}} \frac{r^{n+3}}{\left(1+r^{2}\right)^{n-1}} d r\right. \\
& \left.+\frac{n-2}{2} \int_{0}^{r M_{i}^{\frac{p_{i}-1}{2}}} \frac{r^{n+1}}{\left(1+r^{2}\right)^{n-1}} d r\right) .
\end{aligned}
$$

When $n=6$ and $i$ is large, this last expression is nonnegative because the first integral diverges while the second one is finite. If $n=7$ and $i$ is large, again the expression is nonnegative since

$$
\int_{0}^{\infty} \frac{r^{n+3}}{\left(1+r^{2}\right)^{n-1}} d r=\frac{n+2}{n-6} \int_{0}^{\infty} \frac{r^{n+1}}{\left(1+r^{2}\right)^{n-1}} d r .
$$

To see this, first observe that, if $m+1<2 k$, integration by parts gives

$$
\int_{0}^{\infty} \frac{t^{m}}{\left(1+t^{2}\right)^{k}} d t=\frac{m-1}{2(k-1)} \int_{0}^{\infty} \frac{t^{m-2}}{\left(1+t^{2}\right)^{k-1}} d t .
$$


Since

$$
\int_{0}^{\infty} \frac{t^{m-2}}{\left(1+t^{2}\right)^{k-1}} d t=\int_{0}^{\infty} \frac{t^{m-2}}{\left(1+t^{2}\right)^{k}} d t+\int_{0}^{\infty} \frac{t^{m}}{\left(1+t^{2}\right)^{k}} d t
$$

one gets

$$
\int_{0}^{\infty} \frac{t^{m}}{\left(1+t^{2}\right)^{k}} d t=\frac{m-1}{2 k-m-1} \int_{0}^{\infty} \frac{t^{m-2}}{\left(1+t^{2}\right)^{k}} d t
$$

Just choose $m=n+4$ and $k=n+1$ to obtain (6.17).

The proof is finished by using the inequality (6.14).

q.e.d.

\section{Compactness theorem}

In this section we will prove the a priori estimates for the Yamabe problem in the non-locally conformally flat case, for $4 \leq n \leq 7$.

The next Proposition is fundamental since it allows us to use the symmetry estimates we proved before.

Proposition 7.1. Suppose the blowup $x_{i} \rightarrow \bar{x}$ is isolated, $4 \leq n \leq 7$. Then it is also isolated simple. 6.2

The proof of this statement is just as in 9], based on Proposition

Once we have established Proposition [7.1 again following [9], we have:

TheOREM 7.2. Suppose $u_{i} \in \mathbb{M}_{p_{i}}$ is a sequence satisfying $\max _{M} u_{i} \rightarrow$ $\infty$, as $i \rightarrow \infty$. Then $p_{i} \rightarrow \frac{n+2}{n-2}$. Moreover, after passing to a subsequence,

(1) the set $S=\left\{\right.$ blowup points of $\left.u_{i}\right\}$ is finite;

(2) every blowup point of $u_{i}$ is an isolated simple blowup point.

Now let us turn to the statement and proof of the compactness theorem.

THEOREM 7.3. Let $\left(M^{n}, g\right)$ be a smooth closed Riemannian manifold with positive Yamabe quotient, not conformally equivalent to $\left(\mathbb{S}^{n}, g_{0}\right)$. Assume $4 \leq n \leq 7$. Then, for every $\epsilon>0$, there exists a positive constant $C=C(\epsilon, g)$ so that

$$
\left\{\begin{array}{r}
1 / C \leq u \leq C \text { and } \\
\|u\|_{C^{2, \alpha}(M)} \leq C
\end{array}\right.
$$

for every $u \in \cup_{1+\epsilon \leq p \leq \frac{n+2}{n-2}} \mathbb{M}_{p}$. 
Proof. Standard elliptic estimates and the Harnack inequality imply that it suffices to estimate $\|u\|_{C^{0}(M)}$. Suppose, by contradiction, that $\cup_{1+\epsilon \leq p \leq \frac{n+2}{n-2}} \mathbb{M}_{p}$ is not bounded in $C^{0}(M)$. This means that there exist $1+\epsilon \leq p_{i} \leq \frac{n+2}{n-2}$ and $u_{i} \in \mathbb{M}_{p_{i}}$ with

$$
\max _{M} u_{i} \rightarrow \infty \text { as } i \rightarrow \infty \text {. }
$$

From Theorem 7.2 we know that this is only possible if $p_{i} \rightarrow \frac{n+2}{n-2}$.

Now Theorem 7.2 implies that, after possibly passing to a subsequence, $u_{i}$ has $\mathrm{N}$ isolated simple blow-up points $x_{i}^{(1)} \rightarrow x^{(1)}, \ldots, x_{i}^{(N)} \rightarrow$ $x^{(N)}$, for some integer $\mathrm{N}$.

Define $w_{i}(x)=u_{i}\left(x_{i}^{(1)}\right) u_{i}(x)$. We can suppose, for example, that

$$
u_{i}\left(x_{i}^{(1)}\right)=\min \left\{u_{i}\left(x_{i}^{(1)}\right), \ldots, u_{i}\left(x_{i}^{(N)}\right)\right\} \text { for all } i .
$$

Proposition 4.5 then implies that there exists $\rho, c>0$ such that

$$
w_{i}(x) \leq c d\left(x, x_{i}^{(j)}\right)^{2-n} \text { when } d\left(x, x_{i}^{(j)}\right) \leq \rho, 1 \leq j \leq N .
$$

On the other hand, we know that the sequence $u_{i}$ is uniformly bounded in $M \backslash \cup_{j=1}^{j=N} B_{\frac{\rho}{4}}\left(x^{(j)}\right)$, since there is no blowup point in that region. Then the Harnack inequality implies $w_{i}$ is uniformly bounded in $M \backslash \cup_{j=1}^{j=N} B_{\frac{\rho}{2}}\left(x^{(j)}\right)$. This and inequality (7.1) imply, after passing to a subsequence,

$$
u_{i}\left(x_{i}^{(1)}\right) u_{i}(x) \rightarrow h(x)=\sum_{j=1}^{N} a_{j} G_{x^{(j)}}(x)+b(x)
$$

in $C_{l o c}^{2}\left(M \backslash\left\{x^{(1)}, \ldots, x^{(N)}\right\}\right)$, where $a_{1}, \ldots, a_{N}$ are nonnegative constants, $G_{x^{(j)}}$ is the Green function of the conformal Laplacian with pole at $x^{(j)}$ and $b(x)$ is a regular $C^{2}$ function satisfying $L_{g}(b)=0$ in M. Note that, since the first eigenvalue of the conformal Laplacian $L_{g}$ is positive, $b \equiv 0$.

Since the Green functions considered are positive and $a_{1}>0$ because of the Corollary [4.6, we obtain the following expansion ([7]) :

$$
h(x)=a d\left(x, x^{(1)}\right)^{2-n}+A+O(r), \text { for } 4 \leq n \leq 6
$$

or

$$
h(x)=a d\left(x, x^{(1)}\right)^{2-n}-c R_{; i j} x_{i} x_{j} r^{-3}+A+O(r), \text { for } n=7 .
$$

The Positive Mass Theorem asserts that $A>0$. 
We want to compute the limit

$$
\liminf _{r \rightarrow 0} P^{\prime}(r, h) \text {. }
$$

Since $r^{2-n}$ is harmonic, definition (6.9) gives

$$
P^{\prime}(r, h)=\int_{|x|=r}\left\{-\frac{(n-2)^{2}}{2} a A r^{1-n}+O\left(r^{2-n}\right)\right\} d \sigma_{r} .
$$

When $n=7$, we use symmetry and the fact that $\Delta R(0)=-\frac{1}{6}|W(0)|^{2}=$ 0 .

Then

$$
\liminf _{r \rightarrow 0} P^{\prime}(r, h)=-\frac{(n-2)^{2}}{2} a A \sigma_{n-1}<0,
$$

by the Positive Mass Theorem.

This contradicts Theorem 6.2 and it finishes the proof.

q.e.d.

Fernando C. Marques, Instituto de Matemática Pura e Aplicada (IMPA), Estrada Dona Castorina 110, 22460-320, Rio de Janeiro - RJ, Brazil

Email address : coda@impa.br 



\section{Bibliography}

[1] T. Aubin. Équations différentielles non linéaires et probléme de Yamabe concernant la courbure scalaire. J. Math. Pures Appl., 55:269-296, 1976.

[2] L. Caffarelli, B. Gidas, and J. Spruck. Asymptotic symmetry and local behavior of semilinear elliptic equations with critical Sobolev growth. Comm. Pure Appl. Math, 42:271-297, 1989.

[3] C. C. Chen and C. S. Lin. Estimates of the scalar curvature equation via the method of moving planes. ii. J. Diff. Geom., 49:115-178, 1998.

[4] O. Druet. From one bubble to several bubbles: the low-dimensional case. J. Diff. Geom., 63(3):399-473, 2003.

[5] O. Druet. Compactness for Yamabe metrics in low dimensions. Int. Math. Res. Not., 23:1143-1191, 2004.

[6] Z. C. Han and Y. Y. Li. The Yamabe problem on manifolds with boundary: existence and compactness results. Duke Math. Journal, 99(3):489-542, 1999.

[7] J. Lee and T. Parker. The Yamabe problem. Bull. Amer. Math. Soc., 17:37-91, 1987.

[8] Y. Y. Li. Prescribing scalar curvature on $\mathbb{S}^{n}$ and related problems, part I. $J$. Differential Equations, 120:319-410, 1995.

[9] Y. Y. Li and M. Zhu. Yamabe type equations on three dimensional Riemannian manifolds. Comm. in Contemporary Mathematics, 1(1):1-50, 1999.

[10] F. C. Marques. Existence and compactness theorems on conformal deformation of metrics. PhD thesis, Cornell University, 2003.

[11] M. Obata. The conjectures on conformal transformations of Riemannian manifolds. J. Diff. Geom., 6:247-258, 1971.

[12] D. Pollack. Compactness results for complete metrics of constant positive scalar curvature on subdomains of $\mathbb{S}^{n}$. Indiana Univ. Math. J., 42(4):1441-1456, 1993.

[13] R. Schoen. On the number of constant scalar curvature metrics in a conformal class. In Differential Geometry: A Symposium in Honor of Manfredo do Carmo.

[14] R. Schoen. A report on some recent progress on nonlinear problems in geometry. In Surveys in differential geometry (Cambridge, MA, 1990), pages 201-241. Lehigh Univ., Bethlehem, PA.

[15] R. Schoen. Conformal deformation of a Riemannian metric to constant scalar curvature. J. Differential Geometry, 20:479-495, 1984.

[16] R. Schoen and S. T. Yau. On the proof of the positive mass conjecture in General Relativity. Comm. Math. Phys., 65:45-76, 1979. 
[17] R. Schoen and D. Zhang. Prescribed scalar curvature on the n-sphere. Calc. Var. and PDEs, 4:1-25, 1996.

[18] N. Trudinger. Remarks concerning the conformal deformation of a Riemannian structure on compact manifolds. Ann. Scuola Norm. Sup. Pisa Cl. Sci., 22(3):165-274, 1968.

[19] H. Yamabe. On a deformation of Riemannian structures on compact manifolds. Osaka Math. J., 12:21-37, 1960. 